\title{
Wavelet solution of variable order pseudodifferential equations
}

\section{Journal Article}

Author(s):

Schneider, R.; Reichmann, O.; Schwab, Christoph

Publication date:

2010

Permanent link:

https://doi.org/10.3929/ethz-b-000018365

Rights / license:

In Copyright - Non-Commercial Use Permitted

Originally published in:

Calcolo 47(2), https://doi.org/10.1007/s10092-009-0012-y 


\title{
Wavelet solution of variable order pseudodifferential equations
}

\author{
R. Schneider • O. Reichmann • C. Schwab
}

Received: 19 July 2005 / Accepted: 26 August 2009 / Published online: 15 October 2009

(C) Springer-Verlag 2009

\begin{abstract}
Sobolev spaces $H^{m(x)}(I)$ of variable order $0<m(x)<1$ on an interval $I \subset \mathbb{R}$ arise as domains of Dirichlet forms for certain quadratic, pure jump Feller processes $X_{t} \in \mathbb{R}$ with unbounded, state-dependent intensity of small jumps. For spline wavelets with complementary boundary conditions, we establish multilevel norm equivalences in $H^{m(x)}(I)$ and prove preconditioning and wavelet matrix compression results for the variable order pseudodifferential generators $A$ of $X$.

Sufficient conditions on $A$ to satisfy a Gårding inequality in $H^{m(x)}(I)$ and timeanalyticity of the semigroup $T_{t}$ associated with the Feller process $X_{t}$ are established.

As application, wavelet-based algorithms of log-linear complexity are obtained for the valuation of contingent claims on pure jump Feller-Lévy processes $X_{t}$ with statedependent jump intensity by numerical solution of the corresponding Kolmogoroff equations.
\end{abstract}

Keywords Feller processes - Wavelets · Pseudodifferential operators · Analytic semigroups $\cdot$ Option pricing

Mathematics Subject Classification (2000) 65 N30 $\cdot 35 \mathrm{~K} 15 \cdot 45 \mathrm{~K} 05$

Work supported in part under the IHP Network "Breaking Complexity" under EC contract number HPRN-CT-2002-00286 with support by the Swiss Federal Office for Science and Education under grant No. BBW 02.0418.

R. Schneider

Institut für Mathematik, Technische Universität Berlin, 10623 Berlin, Germany

O. Reichmann · C. Schwab $(\bowtie)$

Seminar für Angewandte Mathematik, Eidgenössische Technische Hochschule, 8092 Zürich, Switzerland

e-mail: christoph.schwab@sam.math.ethz.ch 


\section{Introduction}

Arbitrage-free values $v(x, \bar{t})$ of contingent claims on assets whose log-returns are modeled by a strong Markov process $X_{t}$ with state space $\mathbb{R}$ can be expressed as (conditional) expectations of prices at time of maturity $\bar{t}>0$, i.e.

$$
v(x, \bar{t})=\mathbb{E}^{x}\left(g\left(X_{\bar{t}}\right)\right)
$$

(e.g. [9]). Here $\mathbb{E}^{x}$ denotes expectation with respect to a martingale measure of $X_{t}$, conditional to $X_{0}=x$, and $g(x)$ is a given, so-called payoff function of the contingent claim. The classical example for $X_{t}$ is geometric Brownian motion in the BlackScholes-Merton model [3, 24].

Definition (1.1) suggests to estimate $v(x, \bar{t})$ by Monte-Carlo simulation, i.e. by averaging over $M$ realizations $\left\{X_{t}\left(\omega_{j}\right)\right\}_{j=1}^{M}$ of sample-paths. The statistical error in the estimate $v^{M}(x, \bar{t})$ thus obtained tends to zero not faster than $O(1 / \sqrt{M})$, in general.

Deterministic methods to compute $v(x, \bar{t})$ are based on the semigroup $T_{t}$ of $X_{t}$ defined by

$$
v(x, t)=\left(T_{t} g\right)(x)=\mathbb{E}^{x}\left(g\left(X_{t}\right)\right), \quad t>0 .
$$

The process $X_{t}$ and its Semigroup $T_{t}$ are called Feller if

(i) $T_{t}$ maps $C_{0}(\mathbb{R})$, the continuous functions on $\mathbb{R}$ vanishing at infinity, into itself:

$$
T_{t}: C_{0}(\mathbb{R}) \rightarrow C_{0}(\mathbb{R}) \quad \text { boundedly }
$$

and

(ii) if $T_{t}$ is strongly continuous, i.e. $\lim _{t \rightarrow 0^{+}}\left\|u-T_{t} u\right\|_{L^{\infty}(\mathbb{R})}=0$ for all $u \in C_{0}(\mathbb{R})$.

The infinitesimal generator $A_{X}$ with domain $\mathcal{D}\left(A_{X}\right)$ of a Feller process $X_{t}$ with semigroup $T_{t}$ is defined by the strong limit

$$
A_{X} u:=\lim _{t \rightarrow 0^{+}} \frac{1}{t}\left(T_{t} u-u\right)
$$

on all functions $u \in \mathcal{D}\left(A_{X}\right) \subset C_{0}(\mathbb{R})$ for which the limit (1.3) exists w.r. to the supnorm. We call $\left(A_{X}, \mathcal{D}\left(A_{X}\right)\right)$ Feller generator of $X$. Feller generators admit the positive maximum principle, i.e.

$$
\text { if } \quad u \in \mathcal{D}\left(A_{X}\right) \quad \text { and } \quad \sup _{x \in \mathbb{R}} u(x)=u\left(x_{0}\right)>0, \quad \text { then } \quad\left(A_{X} u\right)\left(x_{0}\right) \leq 0
$$

and admit a pseudodifferential representation (e.g. [7, 13, 14]):

Theorem 1 Let $(A, \mathcal{D}(A))$ be a Feller generator with $C_{0}^{\infty}(\mathbb{R}) \subset \mathcal{D}(A)$. Then $\left.A\right|_{C_{0}^{\infty}(\mathbb{R})}$ is a pseudodifferential operator,

$$
(A u)(x)=-a(x, D) u(x)=-(2 \pi)^{-1 / 2} \int_{\mathbb{R}} a(x, \xi) \hat{u}(\xi) e^{i x \xi} d \xi
$$


for $u \in C_{0}^{\infty}(\mathbb{R})$ with symbol $a(x, \xi): \mathbb{R} \times \mathbb{R} \rightarrow \mathbb{C}$ which is measurable and locally bounded in $(x, \xi)$ and which admits the Lévy-Khintchine representation

$$
\begin{aligned}
a(x, \xi)= & c(x)-i \gamma(x) \xi+(\sigma(x))^{2} \xi^{2} \\
& +\int_{0 \neq y \in \mathbb{R}}\left(1-e^{i y \xi}+\frac{i y \xi}{1+y^{2}}\right) N(x, d y) .
\end{aligned}
$$

The parameters $c(x), \gamma(x), \sigma(x), N(x, d y)$ in (1.6) are called characteristics of the Feller process $X_{t}$. Spatially and temporally homogeneous Feller processes $X_{t}$ are Lévy-processes (e.g. [2, 6, 29]). Their characteristics, the Lévy characteristics, do not depend on $x$ explicitly.

Symbols $a(x, \xi)$ of the form (1.6) are called negative definite symbols. In (1.6), $N(x, d y)$ is the compensated jump measure of the Feller process $X_{t}^{x}=\left\{X_{t} \mid X_{0}=x\right\}$ which satisfies

$$
\sup _{x \in \mathbb{R}} \int_{\mathbb{R}} \min \left(1, y^{2}\right) N(x, d y)<\infty .
$$

Fast deterministic computation of the conditional expectation $v(x, t)$ in (1.2) across all maturities $0<t<\bar{t}$ is based on the numerical solution of the backward Kolmogoroff equation

$$
v_{t}+A v=0,\left.\quad v\right|_{t=T}=g .
$$

In the Black-Scholes model [3], $X_{t}$ is a diffusion for which $N(x, d y)=0$ in (1.6). The generator $A$ in (1.8) is a diffusion with possibly spatially inhomogeneous characteristics $c(x), \gamma(x), \sigma(x)$, resp. killing, drift and volatility, corresponding to local volatility models.

Exploiting the analyticity of the semigroup $T_{t}$ and a spline wavelet discretization of degree $p \geq 1$ for generators $A$ in (1.5) which are classical pseudodifferential operators of constant order $2 m, 0 \leq m \leq 1$, it has been shown in [21-23] that $v(x, t)$ can be computed for $0<t \leq T$ with essentially $O(N)$ work and memory essentially ${ }^{1}$ to accuracy $O\left(N^{-p-1}\right)$. Key ingredient in the numerical analysis in [22, 23] were (i) wavelet norm equivalences in the "energy" space of $A$ (which are also crucial ingredients for adaptive solution methods), and (ii) the wavelets' vanishing moment property to compress the $N^{2}$ entries in the stiffness matrices of $A$ to $O(N \log N)$ "essential" elements without loss in accuracy.

Here, we address the efficient solution of (1.8) for pure jump processes $X$ with state dependent jump intensity. In this case, $\sigma=0$ in (1.6) and the domain $V$ of the Dirichlet form associated to $A_{X}$ is a Sobolev space of variable order $m(x) \in(0,1)$. Development of linear-complexity solvers for the Kolmogoroff equation (1.8) along the lines of $[22,23]$ and also for the pseudodifferential inequality arising from expectations over the stopped processes $X$ (e.g. [20]) requires (a) verification of wavelet norm equivalences in the "energy" space $H^{m(x)}$ and (b) wavelet compression of the generator $A_{X}$ or, equivalently, of the jump measure of the Feller process $X$ and (c)

\footnotetext{
${ }^{1}$ Throughout, "essentially" means up to powers of $\log N$ or $\log h$ which will be clear from the context.
} 
establishing time-analyticity of the semigroup $T_{t}$. Proving (a)-(c) is the main purpose of the present paper.

Its outline is as follows: in Sect. 2, we introduce a class of pseudodifferential operators of variable order containing, in particular, certain generators $A_{X}$ of the form (1.6). To analyze wavelet-based solution algorithms for (1.8), we derive, based on the calculus of $[12,18]$, estimates for the Schwartz kernels of $A_{X}$, resp. the densities of the compensated jump measure $N_{X}(x, d y)$ of $X_{t}$ with respect to the Lebesgue measure $d y$ which are interesting in their own right. In Sect. 3, we define the variable order Sobolev spaces $H^{m(x)}$ for $0 \leq m(x)<1$ which are the domains of Dirichlet forms of $A_{X}$. They also form the basis of Galerkin discretization of $A_{X}$. In Sect. 4, we use the bounds on the Schwartz kernels to establish our main results: multilevel norm equivalences in the variable order spaces $H^{m(x)}$ for $0 \leq m(x)<1$ and compression estimates for the moment matrices of $A_{X}$ in the wavelet basis. In Sect. 5, we prove the Gårding inequality for the generator (1.6) in variable order Sobolev spaces and deduce time-analyticity of the Semigroup $T_{t}$. The final Sect. 6 addresses the generalization of the fast pricing algorithms from $[22,23]$ to variable order generators.

\section{Generators of variable intensity Feller-Lévy processes}

The infinitesimal generator $A_{X}$ of the Feller process $X$ is the pseudodifferential operator $a(x, D)$ provided by (1.5) in Theorem 1 . Denote by $K_{X}(x, y)$ the Schwartz kernel of the nonlocal part of $A_{X}$. The distribution $K_{X}(x, y)$ is the density of the jump measure $N_{X}(x, d y)$ of $X$ with respect to the Lebesgue measure $d y$, i.e.

$$
N_{X}(x, d y)=K_{X}(x, y) d y .
$$

For pure jump Feller processes $X$ with state-dependent jump-intensity, domains $\mathcal{D}\left(A_{X}\right)$ of their generators $A_{X}$ are Sobolev spaces of variable order $m(x)$ which, by (1.7), satisfies $m(x)<1$. Wavelet solution of the derivative pricing pseudodifferential equation (1.8) for variable intensity processes $X$ with generators of type (1.6), (1.7) requires multilevel norm equivalences. To prove these, we use a calculus for variable order pseudodifferential operators. We start by identifying classes of variable intensity pure jump Feller processes $X$ through conditions on the Lévy symbols $a(x, \xi)$ in (1.6) of their infinitesimal generators $A_{X}$.

Throughout we use the notation $\langle\xi\rangle:=\left(1+|\xi|^{2}\right)^{1 / 2}$. We define a class of quadratic, pure jump Feller processes $X$ with variable jump intensity through their generators $A_{X}$ which are variable order pseudodifferential operators. They, in turn, are given in terms of their symbols. The following symbol classes of variable order have been introduced in [16].

Definition 1 Let $0 \leq \delta<\rho \leq 1$ and let $m(x) \in C^{\infty}(\mathbb{R})$ be a real-valued function all of which derivatives are bounded on $\mathbb{R}$. The symbol $a(x, \xi)$ belongs to the class $S_{\rho, \delta}^{m(x)}$ of symbols of variable order $m(x)$ if $a(x, \xi) \in C^{\infty}(\mathbb{R} \times \mathbb{R})$ and $m(x)=s+\tilde{m}(x)$ with $\tilde{m} \in \mathcal{S}(\mathbb{R})$ a tempered function, and if, for every $\alpha, \beta \in \mathbb{N}_{0}$ there is a constant $c_{\alpha, \beta}$ such that

$$
\forall x, \xi \in \mathbb{R}: \quad\left|D_{x}^{\beta} D_{\xi}^{\alpha} a(x, \xi)\right| \leq c_{\alpha, \beta}\langle\xi\rangle^{m(x)-\rho|\alpha|+\delta|\beta|} .
$$


The variable order pseudo-differential operators $A(x, D) \in \Psi_{\rho, \delta}^{m(x)}$ correspond to symbols $a(x, \xi) \in S_{\rho, \delta}^{m(x)}$ by

$$
A(x, D) u(x):=\frac{1}{2 \pi} \int_{\mathbb{R}} \int_{\mathbb{R}} e^{i(x-y) \cdot \xi} a(x, \xi) u(y) d y d \xi, \quad u \in C_{0}^{\infty}(\mathbb{R}) .
$$

Given a variable order $m(x)$, we define

$$
\bar{m}:=\sup _{x \in \mathbb{R}} m(x), \quad \underline{m}:=\inf _{x \in \mathbb{R}} m(x)
$$

and assume $0 \leq \delta<\rho \leq 1$ throughout.

For $a(x, \xi) \in S_{\rho, \delta}^{m(x)}$, the Martingale Problem is the problem of existence of a Feller Process $X$ with given generator $A(x, D)$. We have ([12, 16], [14, Chap. 2.10] and [30]).

Proposition 1 For every negative definite symbol $a(x, \xi) \in S_{\rho, \delta}^{m(x)}$ of variable order $m(x)$ with $1 \geq \rho>\delta>0$ exists a unique Feller process $X_{t}$ with generator $A_{X}$ as in (1.5), (1.6) and jump measure $N_{X}(x, d y)$ as in (2.1).

By $F P_{\rho, \delta}^{m(x)}$ we denote the set of all Feller processes with generator $A_{X} \in \Psi_{\rho, \delta}^{m(x)}$ given by a negative definite characteristic function $a(x, \xi) \in S_{\rho, \delta}^{m(x)}$. Their domains $\mathcal{D}\left(A_{X}\right)$ are Sobolev spaces of variable order. Our definition and characterization of these variable order Sobolev spaces will be based on variable order Riesz potentials $\Lambda^{m(x)}$ with symbol $a(x, \xi)=\langle\xi\rangle^{m(x)}$. The operators $\Lambda^{m(x)}$ are the generators of so-called "stable-like" Feller processes whose existence has been established in [1]. More general pseudodifferential generators and their associated Feller processes have been considered for example in $[12,16,30]$. Since $\left|D_{x}^{\beta} D_{\xi}^{\alpha}\langle\xi\rangle^{m(x)}\right| \lesssim$ $\langle\xi\rangle^{m(x)-|\alpha|}|\log \langle\xi\rangle|^{|\beta|}$, we have $a_{\Lambda} \in S_{\rho, \delta}^{m(x)} \subset S_{\rho, \delta}^{\bar{m}}$ for all $0<\delta<\rho \leq 1$. The following lemma from [16] is the variable order analogue to a classical result for constant order pseudo-differential operators (see, e.g., Theorems 3.1 to 3.4 in [33] or Chap. VI.6 in [34]).

Lemma 1 Consider compound symbols of the form $a(x, \xi, y) \in C^{\infty}(\mathbb{R} \times \mathbb{R} \times \mathbb{R})$ satisfying

$$
\left|D_{x}^{\alpha} D_{\xi}^{\beta} D_{y}^{\gamma} a(x, \xi, y)\right| \leq c_{\alpha, \beta, \gamma}\langle\xi\rangle^{m(x)+\delta(|\alpha|+|\gamma|)-\rho|\beta|},
$$

together with the corresponding operators

$$
(A(x, D, y) u)(x):=\frac{1}{2 \pi} \int_{\mathbb{R}} \int_{\mathbb{R}} e^{i(x-y) \xi} a(x, \xi, y) u(y) d y d \xi,
$$

$u \in C_{0}^{\infty}(\mathbb{R})$. If $0 \leq m(x)$ for all $x \in \mathbb{R}$ and $\|m\|_{L^{\infty}(\mathbb{R})}<1$, and $A(x, D, y)$ is compactly supported (see e.g. [17, 33]), there exists a pseudo-differential operator $B \in \Psi_{\rho, \delta}^{m(x)}$ with symbol $b \in S_{\rho, \delta}^{m(x)}$ together with a pseudo-differential operator 
$C \in \Psi_{\rho, \delta}^{m(x)-\rho+\delta}$, such that

$$
A(x, D, y)=B(x, D)+C
$$

holds.

Furthermore there exist pseudo-differential operators $T_{1}, T_{2} \in \Psi_{1, \delta}^{-\epsilon}, \delta>0$, and an $\epsilon>0$, such that, for $a(x, \xi):=\langle\xi\rangle^{m(x)} \in S_{1, \delta}^{m(x)}, b(x, \xi):=\langle\xi\rangle^{-m(x)} \in S_{1, \delta}^{-m(x)}$ and corresponding operators $A(x, D) \in \Psi_{1, \delta}^{m(x)}, B(x, D) \in \Psi_{1, \delta}^{-m(x)}$ there holds

$$
A(x, D) B(x, D)=I+T_{1}, \quad B(x, D) A(x, D)=I+T_{2} .
$$

A calculus for variable order pseudodifferential operators $\Psi_{\rho, \delta}^{m(x)}$ was developed in [16], see also [12].

Define the operators $L$ by

$$
(L u)(x):=\left(\Lambda^{m(x)} u\right)(x):=\frac{1}{2 \pi} \iint e^{i \xi(x-y)}\langle\xi\rangle^{m(y)} u(y) d y d \xi .
$$

Then the calculus [16] shows that $L^{\top}:=\Lambda^{m(x)} \in \Psi_{1, \delta}^{m(x)}$ and the operators $A=L$, $A=L^{\top} \in \Psi_{1, \delta}^{m(x)}, A=L^{-1} \in \Psi_{1, \delta}^{-m(x)}$ and $A=L L^{\top}=\left(\Lambda^{m(x)}\right)^{\top}\left(\Lambda^{m(x)}\right) \in \Psi_{1, \delta}^{2 m(x)}$, as well as $A^{(-1)}=\left(L L^{\top}\right)^{(-1)}=\left(\Lambda^{-m(x)}\right)^{\top}\left(\Lambda^{-m(x)}\right) \in \Psi_{1, \delta}^{-2 m(x)}$ can be expressed as singular integrals with respect to their Schwartz kernels $K_{A}(x, y)$ (related to the jump measure $N_{X}(x, d y)$ of the Markov process $X$ with generator $A$ by (2.1))

$$
A u(x)=\int_{\mathbb{R}} K_{A}(x, y) u(y) d y .
$$

Formally, these kernels are given by oscillatory integrals

$$
K_{A}(x, y)=\frac{1}{2 \pi} \int_{\mathbb{R}} e^{i(x-y) \cdot \xi} a_{A}(x, \xi, y) d \xi,
$$

with

$$
a_{L}(x, \xi)=\langle\xi\rangle^{m(x)}, \quad a_{L L^{\top}}(x, \xi, y)=\langle\xi\rangle^{m(x)+m(y)}
$$

and

$$
a_{L^{-1}}(x, \xi, y)=\langle\xi\rangle^{-m(x)}+b(x, \xi, y)
$$

with a corresponding pseudo-differential operator $B \in \Psi_{1, \delta}^{-\epsilon}$ for sufficiently small $\epsilon>0$.

The following lemma plays a crucial role in the wavelet compression of $a_{L}(x, D)$.

Lemma 2 For any $0<\delta<1$, the Schwartz kernel $K_{A}(x, y)$ of $A=L L^{\top} \in \Psi_{1, \delta}^{2 m(x)}$ satisfies the Caldéron-Zygmund type estimate

$$
\left|D_{x}^{\alpha} D_{y}^{\beta} K_{A}(x, y)\right| \leq C_{\alpha, \beta, \delta}|x-y|^{-(1+m(x)+m(y)+(1-\delta)(|\alpha|+|\beta|))},
$$


where $x \neq y,|x-y| \lesssim 1$. For $A^{-1}=\left(L L^{\top}\right)^{(-1)} \in \Psi_{1, \delta}^{-2 m(x)}$, a corresponding estimate holds

$$
\left|D_{x}^{\alpha} D_{y}^{\beta} K_{A^{-1}}(x, y)\right| \leq C_{\alpha, \beta, \delta}|x-y|^{-(1-m(x)-m(y)+(1-\delta)(|\alpha|+|\beta|))} .
$$

For $|x-y| \rightarrow \infty$ the kernels are rapidly decaying in the sense that

$$
\left|D_{x}^{\alpha} D_{y}^{\beta} K(x, y)\right| \lesssim|x-y|^{-N}
$$

for every $N>0$ and for $K=K_{A}$ and $K=K_{A^{-1}}$.

The technique for validating of the above assertion is standard, e.g. Chap. VI of [34]. For the reader's convenience we provide a proof. Let $\widehat{\chi} \in C_{0}^{\infty}(\mathbb{R})$ be a cut-off function, $0 \leq \widehat{\chi}(x) \leq 1, \widehat{\chi}(x)=1$ if $x \in[0,1]$, with support supp $\widehat{\chi} \subset[0,2]$, such that $\widehat{\Psi}(\xi)=\widehat{\chi}(\xi)-\widehat{\chi}(2 \xi)$ defines a Littlewood Paley decomposition cf. [34], i.e.

$$
\widehat{\chi}(\xi)+\sum_{j=1}^{\infty} \widehat{\Psi}\left(2^{-j} \xi\right)=1, \quad \forall \xi \in \mathbb{R} .
$$

The extended symbol $a(x, \xi, y)=\left(1+\xi^{2}\right)^{(m(x)+m(y)) / 2}=\langle\xi\rangle^{m(x)+m(y)}$ of $A=L L^{\top}$ can be decomposed into the sum

$$
\begin{aligned}
a(x, \xi, y) & =a_{0}(x, \xi, y)+\sum_{j=1}^{\infty} a_{j}(x, \xi, y) \\
& :=a(x, \xi, y) \widehat{\chi}(\xi)+\sum_{j=1}^{\infty} a(x, \xi, y) \widehat{\Psi}\left(2^{-j} \xi\right) .
\end{aligned}
$$

From this we obtain the corresponding decomposition of the operator

$$
A=A_{0}+\sum_{j=1}^{\infty} A_{j}
$$

Here the operators $A_{j}$ are defined by their Schwartz kernels

$$
k_{j}(x, z, y)=\frac{1}{2 \pi} \int_{\xi \in \mathbb{R}} e^{i \xi \cdot z} a_{j}(x, \xi, y) d \xi, z=x-y .
$$

Lemma 3 Let $(x, z, y) \mapsto k_{j}(x, z, y)$ be the kernel functions defined by (2.11). Then for all $M \geq 0$ and $z \neq 0$ there holds

$$
\left|D_{x}^{\alpha} D_{y}^{\beta} D_{z}^{\gamma} k_{j}(x, z, y)\right| \leq C_{M, \alpha, \beta}|z|^{-M} 2^{j(-M-1-|\gamma|+\delta(|\alpha|+|\beta|)+m(x)+m(y))} .
$$

Proof Due to definition (2.11) partial integration yields

$$
(-z)^{\mu} D_{z}^{\gamma} D_{x}^{\alpha} D_{y}^{\beta} k_{j}(x, z, y)=\frac{1}{2 \pi} \int e^{i \xi \cdot z} D_{\xi}^{\mu}\left[(i \xi)^{\gamma} D_{x}^{\alpha} D_{y}^{\beta} a_{j}(x, \xi, y)\right] d \xi .
$$


We observe that the integrand is supported on $\left\{\xi: 2^{j-1} \leq|\xi| \leq 2^{j+1}\right\}$ and the volume of this support is bounded by $c 2^{j}$. Therefore, we can estimate the above integral (2.13) directly using

$$
\left|D_{x}^{\alpha} D_{y}^{\beta} D_{\xi}^{\mu} a_{j}(x, \xi, y)\right| \lesssim\langle\xi\rangle^{m(x)+m(y)-|\mu|+\delta(|\alpha|+|\beta|)},
$$

by

$$
\begin{aligned}
\left|z^{\mu} D_{x}^{\alpha} D_{y}^{\beta} D_{z}^{\gamma} k_{j}(x, z, y)\right| & \leq \frac{1}{2 \pi} \int\left|D_{\xi}^{\mu}\left[\xi^{\gamma} D_{x}^{\alpha} D_{y}^{\beta} a_{j}(x, \xi, y)\right]\right| d \xi \\
& \leq C_{\mu, \alpha, \beta} 2^{j(1+m(x)+m(y)+|\gamma|-|\mu|+\delta(|\alpha|+|\beta|))} .
\end{aligned}
$$

Choosing $M=|\mu|$ gives the desired result.

We can now prove Lemma 2.

\section{Proof}

Since

$$
K(x, y)=k_{0}(x, x-y, y)+\sum_{j=1}^{\infty} k_{j}(x, x-y, y)
$$

it is sufficient to estimate

$$
D_{x}^{\alpha} D_{y}^{\beta} k_{0}(x, x-y, y)+D_{x}^{\alpha} D_{y}^{\beta} \sum_{j=1}^{\infty} k_{j}(x, x-y, y) .
$$

From Lemma 3 we conclude the estimate

$$
\begin{aligned}
\left|D_{x}^{\alpha} D_{y}^{\beta} k_{j}(x, x-y, y)\right| \leq & C_{M, \alpha, \beta}|x-y|^{-M} \\
& \times 2^{j(M+1-(1-\delta)(|\alpha|+|\beta|)+m(x)+m(y))} .
\end{aligned}
$$

First we consider the case $|z|=|x-y| \leq 1$ and decompose the sum (2.14)

$$
k_{0}+\sum_{j=1}^{\infty} k_{j}=\sum_{2^{j} \leq|z|^{-1}} k_{j}+\sum_{2^{j} \geq|z|^{-1}} k_{j}
$$

Setting $M=0$ in Lemma 3 the first sum can be majorized by a multiple of

$$
\sum_{2^{j} \leq z^{-1}} 2^{j(1+m(x)+m(y)-(1-\delta)(|\alpha|+|\beta|))} \lesssim|z|^{-(1+m(x)+m(y)-(1-\delta)(|\alpha|+|\beta|))}
$$

provided that $1+m(x)+m(y)-(1-\delta)(|\alpha|+|\beta|)>0$.

To estimate the second sum $\sum_{2^{j} \geq|z|^{-1}} k_{j}$ we choose

$$
M>1+m(x)+m(y)-(1-\delta)(|\alpha|+|\beta|)
$$


and obtain an upper bound

$$
\begin{aligned}
& C|z|^{M} \sum_{2^{j} \geq|z|^{-1}} 2^{-j(1-M+m(x)+m(y)-(1-\delta)(|\alpha|+|\beta|))} \\
& \lesssim|z|^{-((1+m(x)+m(y)-(1-\delta)(|\alpha|+|\beta|))} .
\end{aligned}
$$

To conclude the proof we consider $|z| \geq 1$. Then Lemma 3 provides, for all $N:=M-$ $m(x)+m(y)-(1-\delta)(|\alpha|+|\beta|)>0$, that (2.14) can be bounded by $C_{N, \alpha, \beta}|z|^{-N}$.

Using analogous arguments as above, we obtain

Theorem 2 Let $A \in \Psi_{\rho, \delta}^{2 m(x)}$ for some $0<\delta<\rho \leq 1$. Then the Schwartz-kernel $K_{A}(x, y)$ of A satisfies for all $\alpha, \beta \in \mathbb{N}_{0}$ and all $x, y \in \mathbb{R}$ with $x \neq y$

$$
\left|D_{x}^{\alpha} D_{y}^{\beta} K_{A}(x, y)\right| \leq C_{\alpha, \beta, \delta}|x-y|^{-(1+m(x)+m(y)+(\rho-\delta)(|\alpha|+|\beta|))} .
$$

In the applications we have in mind, the spatial domain is the unit interval $I=$ $(0,1)$ rather than all of $\mathbb{R}$ and the variable order $m(x)$ is only defined on $I$. There holds $0<m(x)<1$, for all $x \in[0,1]$. For technical purposes we extend $m(x)=$ $s+\tilde{m}(x)$ such that there exists $\epsilon>0$ with $\epsilon<m(x)<1-\epsilon$ and $\tilde{m} \in \mathcal{S}(\mathbb{R})$.

\section{Variable order Sobolev spaces}

We define the Sobolev space $H^{m(x)}(\mathbb{R})$ of variable order $m(x) \in[0,1)$ on $\mathbb{R}$ through the $H^{m(x)}(\mathbb{R})$ Sobolev norm given by

$$
\|u\|_{H^{m(x)}(\mathbb{R})}^{2}:=\left\|\Lambda^{m(x)} u\right\|_{L_{2}(\mathbb{R})}^{2}+\|u\|_{L_{2}(\mathbb{R})}^{2} .
$$

We also introduce the inner product

$$
\langle u, v\rangle_{m(x)}:=\left\langle\Lambda^{m(x)} u, \Lambda^{m(x)} v\right\rangle+\langle u, v\rangle,
$$

so that $\langle u, u\rangle_{m(x)}=\|u\|_{H^{m(x)}(\mathbb{R})}^{2}$. The space $H^{-m(x)}(\mathbb{R})$ is defined as the dual space of $H^{m(x)}(\mathbb{R})$ with respect to the $\langle.$, . $\rangle$-duality. An equivalent norm in $H^{-m(x)}(\mathbb{R})$ is given by

$$
\begin{aligned}
\langle u, v\rangle_{-m(x)} & :=\left\langle\Lambda^{m(x)} u, \Lambda^{m(x)} v\right\rangle+\left\langle\Lambda^{-2 \bar{m}} u, v\right\rangle, \\
\|u\|_{H^{-m(x)}(\mathbb{R})}^{2} & =\langle u, u\rangle_{-m(x)} .
\end{aligned}
$$

We remark that due to the symbolic calculus in [16] referred to in Lemma 1, an equivalent definition used by $[16,18]$ is

$$
\|u\|_{H^{m(x)}(\mathbb{R})}:=\left\|\left(\Lambda^{m(x)}\right)^{\top} u\right\|_{L_{2}(\mathbb{R})} .
$$


On a bounded interval $I=(a, b) \subset \mathbb{R}$ we define for a variable order $0 \leq m(x)<1$, $a \leq x \leq b$ the space

$$
\tilde{H}^{m(x)}(I):=\left\{\left.u\right|_{I}\left|u \in H^{m(x)}(\mathbb{R}), u\right|_{\mathbb{R} \backslash \bar{I}}=0\right\} .
$$

It coincides with the closure of $C_{0}^{\infty}(I)$ (the space of smooth functions with support compactly contained in $I$ ) with respect to the norm

$$
\|u\|_{\tilde{H}^{m(x)}(I)}:=\|\tilde{u}\|_{H^{m(x)}(\mathbb{R})},
$$

where $\tilde{u}$ is the zero extension of $u$ to all of $\mathbb{R}$. It follows from Lemma 2 applied to $L=\Lambda^{m(x)}$ and from the positive definiteness of the Dirichlet form of the variable order Riesz potential $\Lambda^{m(x)}$ on $C_{0}^{\infty}(\mathbb{R})$ that for $0<\underline{m} \leq m(x) \leq \bar{m}<1$ an intrinsic norm on $\tilde{H}^{m(x)}(I)$ is given by

$$
\begin{aligned}
\|u\|_{\tilde{H}^{m(x)}(I)}^{2} & :=\|u\|_{L^{2}(I)}^{2}+|u|_{\tilde{H}^{m(x)}(I)}^{2}, \\
|u|_{\tilde{H}^{m(x)}(I)}^{2} & :=\int_{\mathbb{R}} \int_{\mathbb{R}} \frac{|\tilde{u}(x)-\tilde{u}(y)|^{2}}{|x-y|^{1+m(x)+m(y)}} d y d x .
\end{aligned}
$$

Evidently, $\tilde{H}^{m(x)}(I) \subset H^{m(x)}(\mathbb{R})$. We also define $H^{m(x)}(I)=\{u \in \mathcal{D}(I)$ : there exists an extension $\left.l u \in H^{m(x)}(\mathbb{R}),\left.l u\right|_{I}=u\right\}$ supplied with the quotient norm $\|u\|_{H^{m(x)}(I)}:=\inf \left\{\|l u\|_{m(x)}:\left.l u\right|_{I}=u\right\}$. Then $H^{m(x)}(I)$ is isomorphic to the quotient space $H^{m(x)}(\mathbb{R}) / \widetilde{H}^{m(x)}(\mathbb{R} \backslash I)$.

Spaces of order $m(x) \leq 0$ are defined by duality

$$
\left(\widetilde{H}^{m(x)}(I)\right)^{\prime}=H^{-m(x)}(I) \text { and } \quad\left(H^{m(x)}(I)\right)^{\prime}=\widetilde{H}^{-m(x)}(I) .
$$

We remark that if $m(a)>\frac{1}{2}$ then functions $u \in \widetilde{H}^{m(x)}(I)$ have zero Dirichlet trace, i.e. $u(a)=0$.

\section{Spline wavelets with complementary boundary conditions}

Since we discretize the parabolic equation $(1.8)$ in $(0, T) \times I$ in the spatial variable with spline wavelet bases for $V=\tilde{H}^{m(x)}(I)$, we briefly recapitulate basic definitions and results on wavelets from e.g. [5] and the references there. For specific spline wavelet constructions on a bounded interval $I$, we refer to e.g. [10, 27, 35].

The primal wavelet bases $\psi_{l, k}$ span finite dimensional spaces

$$
\mathcal{W}^{l}:=\operatorname{span}\left\{\psi_{l, k}: k \in \nabla_{l}\right\}, \quad \mathcal{V}^{L}:=\bigoplus_{l=-1}^{L-1} \mathcal{W}^{l}, \quad l=-1,0,1 \ldots,
$$

and the dual spaces are defined analogously in terms of the dual wavelets $\tilde{\psi}_{l, k}$ by

$$
\tilde{\mathcal{W}}^{l}:=\operatorname{span}\left\{\tilde{\psi}_{l, k}: k \in \nabla_{l}\right\}, \quad \tilde{\mathcal{V}}^{L}:=\bigoplus_{l=-1}^{L-1} \tilde{\mathcal{W}}^{l}, \quad l=-1,0,1 \ldots
$$


In the sequel we will require the following properties of these functions

1. Biorthogonality: the basis functions $\psi_{l, k}, \tilde{\psi}_{l, k}$ satisfy

$$
\left\langle\psi_{l, k}, \tilde{\psi}_{l^{\prime}, k^{\prime}}\right\rangle=\delta_{l, l^{\prime}} \delta_{k, k^{\prime}}
$$

2. Local support: the diameter of the support is proportional to a meshsize $2^{-l}$,

$$
\text { diam supp } \psi_{l, k} \lesssim 2^{-l}, \quad \text { diam supp } \tilde{\psi}_{l, k} \sim 2^{-l} .
$$

3. Conformity: the basis functions should be sufficiently regular, i.e.

$$
\mathcal{W}^{l} \subset \tilde{H}^{1}(I), \quad \tilde{\mathcal{W}}^{l} \subset H^{\delta}(I) \quad \text { for some } \delta>0, l \geq-1 .
$$

Furthermore $\bigoplus_{l=-1}^{\infty} \mathcal{W}^{l}, \bigoplus_{l=-1}^{\infty} \tilde{\mathcal{W}}^{l}$ are supposed to be dense in $L_{2}(I)$

4. Vanishing moments: The primal basis functions $\psi_{l, k}$ are assumed to satisfy vanishing moment conditions up to order $p^{*}+1 \geq p$

$$
\left\langle\psi_{l, k}, x^{\alpha}\right\rangle=0, \quad \alpha=0, \ldots, d=p^{*}+1, l \geq 0,
$$

and for all dual wavelets, except the ones at each end point, one has

$$
\left\langle\tilde{\psi}_{l, k}, x^{\alpha}\right\rangle=0, \quad \alpha=0, \ldots, d=p+1, l \geq 0 .
$$

At the end points the dual wavelets satisfy only

$$
\left\langle\tilde{\psi}_{l, k}, x^{\alpha}\right\rangle=0, \quad \alpha=1, \ldots, d=p+1, l \geq 0 .
$$

On $I=(0,1)$ we set $\nabla_{l}=\left\{k=1, \ldots, M_{l}\right\}$, where usually $M_{l}=2^{l}$. We remark, that the third condition implies zero Dirichlet condition, namely $\psi_{l, k}(0)=$ $\psi_{l, k}(1)=0$. To guarantee this condition one has to sacrifice the vanishing moment property of one wavelet, e.g. $\psi_{l, k}, l=0, \ldots$, at each end point 0 or 1 . A systematic and general construction for arbitrary order biorthogonal spline wavelets is presented in [8]. Sufficiently far apart from the end points of $(0,1)$, biorthogonal wavelet (e.g. [5] and the references there) bases are used in this approach. Using biorthogonal wavelets in the case $p=1$, piecewise linear spline wavelets vanishing outside $I=(0,1)$ are obtained by simple scaling. The interior wavelets have two vanishing moments and are obtained from the mother wavelet $\psi(x)$ which takes the values $\left(0,-\frac{1}{6},-\frac{1}{3}, \frac{2}{3},-\frac{1}{3},-\frac{1}{6}, 0,0,0\right)$ at the points $\left(0, \frac{1}{8}, \frac{1}{4}, \frac{3}{8}, \frac{1}{2}, \frac{5}{8}, \frac{3}{4}, \frac{7}{8}, 1\right)$ by scaling and translations: $\psi_{l, k}(x):=2^{l / 2} \psi\left(2^{l-3} x-k+2\right)$ for $2 \leq k \leq 2^{l}-3$ and $l \geq 3$. At the left boundary $k=1$, we use the piecewise linear function $\psi_{\text {left }}$ defined by the nodal values $\left(0, \frac{5}{8}, \frac{-3}{4}, \frac{-1}{4}, \frac{1}{4}, \frac{1}{8}, 0,0,0\right)$ and $\psi_{\text {right }}(x)=\psi_{\text {left }}(1-x)$. For more details we refer to [11].

The following simplified basis functions are shown to be quite efficient for the present applications [23]. They have the advantage of an extremely small support. Nevertheless, the dual wavelets do not permit compact support, but they are exponentially decaying. I.e.,

$$
\left|\tilde{\Psi}_{e}(x)\right| \leq C \exp (-\kappa|x|), \quad \kappa>0, x \in \mathbb{R} .
$$


We emphasize that we do need the local support only of the primal wavelets for matrix compression. Therefore, this property is important for an efficient implementation. But we have assumed local support of the dual wavelets only for simplifying the present proof of the norm equivalence. However it is very likely that the present results remain valid for exponentially decaying dual wavelets.

The biorthogonal wavelets in the case $p=1$ are continuous, piecewise linear spline wavelets vanishing outside $I=(0,1)$ (for general intervals $I=(a, b)$, they are obtained by simple scalings). The interior wavelets have two vanishing moments and are obtained from the mother wavelet $\psi(x)$ taking values $\left(0,-\frac{1}{2}, 1,-\frac{1}{2}, 0\right)$ at $\left(0, \frac{1}{4}, \frac{1}{2}, \frac{3}{4}, 1\right)$ by scaling and translation: $\psi_{l, k}(x):=2^{l / 2} \psi\left(2^{l-1} x-(2 k-1) 2^{-2}\right)$ for $1 \leq k \leq 2^{l}-2$ and $l \geq 2$.

The boundary wavelets are constructed from the continuous, piecewise linear functions $\psi_{*}$, with values $\left(0,1,-\frac{1}{2}, 0\right)$ at $\left(0, \frac{1}{4}, \frac{1}{2}, \frac{3}{4}\right)$, and $\psi^{*}$, taking values $\left(0,-\frac{1}{2}, 1,0\right)$ at $\left(\frac{1}{4}, \frac{1}{2}, \frac{3}{4}, 1\right): \psi_{0}^{l}=\psi_{*}\left(2^{l-1} x\right)$ and $\psi_{l, 2^{l}-1}=2^{l / 2} \psi^{*}\left(2^{l-1} x-2^{l-1}+\right.$ 1). The following results are known for wavelets satisfying the above requirements (e.g., [5]).

For all $v_{h}=\sum_{l=0}^{L-1} \sum_{k=1}^{M^{l}} v_{l, k} \psi_{l, k} \in V_{h}=\mathcal{V}^{L}, h \sim 2^{-L}$, there holds the norm equivalence

$$
\left\|v_{h}\right\|_{\tilde{H}^{s}(I)}^{2} \cong \sum_{l=0}^{L} \sum_{k=1}^{M^{l}}\left|v_{l, k}\right|^{2} 2^{2 l s},
$$

for all $0 \leq s<\frac{3}{2}$. Validity of (4.8) in the variable order spaces $\tilde{H}^{m(x)}(I)$ will be shown in Theorem 3 below. Using the abbreviation $\lambda=(l, k) \in \mathcal{I}:=\{\lambda=(l, k)=$ $\left.l=-1,0,1, \ldots, k=1, \ldots, M_{l}\right\}$ any function $v \in \tilde{H}^{s}(I), 0 \leq s \leq p+1$, and, due to $\tilde{H}^{m}(I) \subset \tilde{H}^{m(x)}(I) \subset \tilde{H}^{\bar{m}}(I)$, any function in $\tilde{H}^{m(x)}(I)$ can be represented in the wavelet series

$$
v=\sum_{l=0}^{\infty} \sum_{k=1}^{M^{l}} v_{l, k} \psi_{l, k}=\sum_{\lambda \in \mathcal{I}} v_{\lambda} \psi_{\lambda} .
$$

This makes it possible to define an approximation operator $Q_{h}: \tilde{H}^{m(x)}(I) \rightarrow V_{h}$, defined by

$$
Q_{h} v=\sum_{l=0}^{L} \sum_{k=1}^{M^{l}} v_{l, k} \psi_{l, k} .
$$

For $0 \leq s<\frac{3}{2} \leq t \leq p+1$, we have the approximation property (e.g. [5])

$$
\left\|v-Q_{h} v\right\|_{\tilde{H}^{s}(I)} \leq C h^{t-s}\|v\|_{H^{t}(I)} .
$$




\section{Matrix compression and norm equivalences}

Consider $\Lambda^{m(x)} \in \Psi_{1, \delta}^{m(x)}$ with symbol $\langle\xi\rangle^{m(x)} \in S_{1, \delta}^{m(x)}$. Then $M:=\left(\Lambda^{m(x)}\right)^{\top} \Lambda^{m(x)}$ is, for $u \in C_{0}^{\infty}(\mathbb{R})$, given by the oscillatory integral

$$
\left(\left(\Lambda^{m(x)}\right)^{\top} \Lambda^{m(x)}\right) u(x):=\frac{1}{2 \pi} \int_{\mathbb{R}} \int_{\mathbb{R}} e^{i \xi(x-y)}\langle\xi\rangle^{m(y)+m(x)} u(y) d y d \xi .
$$

For preconditioning as well as to establish norm equivalences in the spaces $\widetilde{H}^{m(x)}(I)$ of variable order, we will consider the stiffness matrices of the operators $\Lambda^{m(x)}$ in the wavelet basis $\psi_{\lambda}$, i.e.

$$
\mathbf{M}=\left(\left\langle\Lambda^{m(x)} \psi_{\lambda^{\prime}}, \Lambda^{m(x)} \psi_{\lambda}\right\rangle\right)_{\lambda, \lambda^{\prime} \in \mathcal{I}}
$$

and

$$
\mathbf{M}^{(-1)}=\left(\left\langle\Lambda^{-m(x)} \psi_{\lambda^{\prime}}, \Lambda^{-m(x)} \psi_{\lambda}\right\rangle\right)_{\lambda, \lambda^{\prime} \in \mathcal{I}} .
$$

We recall (2.4), $\bar{m}:=\sup _{x \in \mathbb{R}} m(x)$ and $\underline{m}:=\inf _{x \in \mathbb{R}} m(x)$. For $\lambda=(l, k)$, we define the extended support $\Omega_{\lambda}$ of a wavelet basis function $\psi_{\lambda}$ by

$$
\Omega_{\lambda}:=\Omega_{l, k}=\bigcup_{l^{\prime} \geq l}\left\{\operatorname{supp} \psi_{\lambda^{\prime}}: \operatorname{supp} \psi_{\lambda} \cap \operatorname{supp} \psi_{\lambda^{\prime}} \neq \emptyset\right\} .
$$

For $\lambda=(l, k)$ we also define

$$
\underline{m}_{\lambda}:=\inf \left\{m(x): x \in \Omega_{\lambda}\right\} \quad \text { and } \quad \bar{m}_{\lambda}:=\sup \left\{m(x): x \in \Omega_{\lambda}\right\} .
$$

Lemma 4 Let $\psi_{l, k}, \psi_{l^{\prime}, k^{\prime}}$ be two wavelet basis functions with compact support and with $p^{*}+1 \geq 2$ vanishing moments. If $\operatorname{dist}\left(\operatorname{supp} \psi_{l, k}\right.$, supp $\left.\psi_{l^{\prime}, k^{\prime}}\right)>0$, then we have for each $0<\delta<1$ the estimate

$$
\begin{aligned}
& \left|\left\langle\Lambda^{m(x)} \psi_{l, k}, \Lambda^{m(x)} \psi_{l^{\prime}, k^{\prime}}\right\rangle\right| \\
& \quad \leq C_{\delta} 2^{-\left(l+l^{\prime}\right)(1 / 2+d)} \operatorname{dist}\left(\operatorname{supp} \psi_{l, k}, \operatorname{supp} \psi_{l^{\prime}, k^{\prime}}\right)^{-(1+2 \bar{m}+2(1-\delta) d)}
\end{aligned}
$$

for come constant $C_{\delta}>0$ independent of $l, l^{\prime}$ as well as

$$
\begin{aligned}
& \quad\left|\left\langle\Lambda^{-m(x)} \widetilde{\psi}_{l, k}, \Lambda^{-m(x)} \widetilde{\psi}_{l^{\prime}, k^{\prime}}\right\rangle\right| \\
& \quad \leq C_{\delta} 2^{-\left(l+l^{\prime}\right)(1 / 2+d)} \operatorname{dist}\left(\operatorname{supp} \widetilde{\psi}_{l, k}, \operatorname{supp} \widetilde{\psi}_{l^{\prime}, k^{\prime}}\right)^{-(1-2 \underline{m}+2(1-\delta) d)} .
\end{aligned}
$$

Proof We define the operator $A=\left(\Lambda^{m(x)}\right)^{\top} \Lambda^{m(x)}$ with extended symbol

$$
a(x, \xi, y)=\langle\xi\rangle^{m(x)+m(y)}
$$

and denote by $K_{A}(\cdot, \cdot)$ the corresponding Schwartz kernel.

Following standard arguments in wavelet matrix compression (e.g., [5, 31]) we obtain 


$$
\begin{aligned}
& \left|\left\langle\Lambda^{m(x)} \psi_{l, k}, \Lambda^{m(x)} \psi_{l^{\prime}, k^{\prime}}\right\rangle\right| \\
& \quad \lesssim\left|\iint K_{A}(x, y) \overline{\psi_{l, k}(x)} \psi_{l^{\prime}, k^{\prime}}(y) d x d y\right| \\
& \quad \lesssim\left(\operatorname{diam} \operatorname{supp} \psi_{l, k}\right)^{d}\left(\operatorname{diam} \operatorname{supp} \psi_{l^{\prime}, k^{\prime}}\right)^{d}\left(\int\left|\psi_{l, k}(x) d x\right|\right) \\
& \quad \times\left(\int\left|\psi_{l^{\prime}, k^{\prime}}(y) d y\right|\right) \sup _{x \in \operatorname{supp} \psi_{l, k} y \in \operatorname{supp} \psi_{l^{\prime}, k^{\prime}}\left|D_{x}^{d} D_{y}^{d} K_{A}(x, y)\right|} \sup ^{\lesssim 2^{-\left(l+l^{\prime}\right)(1 / 2+d)} \operatorname{dist}\left(\operatorname{supp} \psi_{l, k}, \operatorname{supp} \psi_{l^{\prime}, k^{\prime}}\right)^{-(1+2(1-\delta) d+2 \bar{m})} .}
\end{aligned}
$$

In the last step, we have used the fact that $A \in \Psi_{1, \delta}^{2 m(x)} \subset \Psi_{1, \delta}^{2 \bar{m}}$ together with the Caldérón Zygmund property of $K_{A}(x, y)$ shown in Lemma 2. Since $B=$ $\left(\Lambda^{-m(x)}\right)^{\top} \Lambda^{-m(x)} \in \Psi_{1, \delta}^{-2} \underline{m}$ a similar argument proves (5.5).

Since the boundary wavelets of the dual wavelet system do not have vanishing moments, we treat the corresponding matrix coefficients separately.

Lemma 5 If among $\widetilde{\psi}_{l, k}, \widetilde{\psi}_{l^{\prime}, k^{\prime}}$ only the latter wavelet has no vanishing moments, and if

$$
\operatorname{dist}\left(\operatorname{supp} \widetilde{\psi}_{l, k}, \operatorname{supp} \widetilde{\psi}_{l^{\prime}, k^{\prime}}\right)>0
$$

then we have for each $0<\delta<1$ the estimate

$$
\begin{aligned}
& \left|\left\langle\Lambda^{-m(x)} \widetilde{\psi}_{l, k}, \Lambda^{-m(x)} \widetilde{\psi}_{l^{\prime}, k^{\prime}}\right\rangle\right| \\
& \quad \leq C_{\delta} 2^{-l(1 / 2+d)} \operatorname{dist}\left(\operatorname{supp} \psi_{l, k}, \operatorname{supp} \psi_{l^{\prime}, k^{\prime}}\right)^{-(1-2 \underline{m}+(1-\delta) d)} .
\end{aligned}
$$

We need an estimate treating the case that the supports of two wavelet functions overlap. First we investigate the diagonal matrix entries.

Lemma 6 Let $0 \leq m(x), x \in \mathbb{R}$ and $\underline{m}_{\lambda}, \bar{m}_{\lambda}, \lambda=(l, k)$ as defined in (5.4), then

$$
2^{2 l \underline{m}_{\lambda}} \lesssim\left|\left\langle\Lambda^{m(x)} \psi_{l, k}, \Lambda^{m(x)} \psi_{l, k}\right\rangle\right| \lesssim 2^{2 l \bar{m}_{\lambda}}
$$

For $\Lambda^{-1}$ holds correspondingly

$$
2^{-2 l \bar{m}_{\lambda}} \lesssim\left|\left\langle\Lambda^{-m(x)} \widetilde{\psi}_{l, k}, \Lambda^{-m(x)} \widetilde{\psi}_{l, k}\right\rangle\right| \lesssim 2^{-2 l \underline{m}_{\lambda}} .
$$

Proof Observing $\langle\xi\rangle^{m(x)}\langle\xi\rangle^{-\bar{m}} \in S_{1, \delta}^{0}$ for any $\delta>0$, and since $\Lambda^{m(x)}: \tilde{H}^{m(x)}(I) \rightarrow$ $L^{2}(I)$ is one-to-one [18], we estimate

$$
\begin{aligned}
\left\langle\Lambda^{m(x)} \psi_{l, k}, \Lambda^{m(x)} \psi_{l, k}\right\rangle & =\left\|\Lambda^{m(x)} \psi_{l, k}\right\|_{L^{2}(I)}^{2} \lesssim\left\|\Lambda^{\bar{m}_{\lambda}} \psi_{l, k}\right\|_{L^{2}(I)}^{2} \\
& \lesssim \int_{\xi}\left|\int_{x} e^{-i x \xi}\langle\xi\rangle^{\bar{m}_{\lambda}} \psi_{\lambda}(x) d x\right|^{2} d \xi \lesssim 2^{2 l \bar{m}_{\lambda}} .
\end{aligned}
$$


By similar arguments we obtain using $\inf \left\{\theta(x): x \in \Omega_{\lambda}\right\} \geq \underline{m}_{\lambda}$ the estimates

$$
\begin{aligned}
\left\langle\Lambda^{-m(x)} \tilde{\psi}_{l, k}, \Lambda^{-m(x)} \tilde{\psi}_{l, k}\right\rangle & =\frac{1}{2 \pi} \int_{\xi}\left|\int_{x} e^{-i x \xi}\langle\xi\rangle^{-m(x)} \widetilde{\psi}_{l, k}(x) d x\right|^{2} d \xi \\
& \lesssim\left\|\Lambda^{-\underline{m}_{\lambda}} \widetilde{\psi}_{l, k}\right\|_{0}^{2} \lesssim 2^{-2 l \underline{m}_{\lambda}} .
\end{aligned}
$$

We get also lower bounds. For some $C>0$ independent of $\lambda$ holds

$$
\begin{aligned}
\left\langle\Lambda^{m(x)} \psi_{l, k}, \Lambda^{m(x)} \psi_{l, k}\right\rangle & =\frac{1}{2 \pi} \int_{\xi}\left|\int_{x} e^{-i x \xi}\langle\xi\rangle^{m(x)} \psi_{l, k}(x) d x\right|^{2} d \xi \\
& \geq\left\|\Lambda^{-\underline{m}_{\lambda}} \psi_{l, k}\right\|_{0}^{2} \geq C 2^{2 l \underline{m}_{\lambda}} .
\end{aligned}
$$

Similar arguments prove the remaining inequality.

Lemma 7 In case $\operatorname{supp} \psi_{\lambda} \cap \operatorname{supp} \psi_{\lambda^{\prime}} \neq \emptyset$ and $l \leq l^{\prime}$, the following estimate holds for $0 \leq s+\bar{m}_{\lambda}<\gamma$,

$$
\left|\left\langle\Lambda^{m(x)} \psi_{l, k}, \Lambda^{m(x)} \psi_{l^{\prime}, k^{\prime}}\right\rangle\right| \lesssim 2^{-\left|l-l^{\prime}\right| s} 2^{l \bar{m}_{\lambda}+l^{\prime} \bar{m}_{\lambda^{\prime}}} .
$$

Proof We suppose that $l \geq l^{\prime}$. Then, for $0 \leq s<\gamma-\bar{m}$,

$$
\begin{aligned}
\left|\left\langle\Lambda^{m(x)} \psi_{l, k}, \Lambda^{m(x)} \psi_{l^{\prime}, k^{\prime}}\right\rangle\right| & \leq\left\|\Lambda^{m(x)-s} \psi_{l, k}\right\|_{0}\left\|\Lambda^{m(x)+s} \psi_{l^{\prime}, k^{\prime}}\right\|_{0} \\
& \lesssim\left\|\Lambda^{\bar{m}_{\lambda^{\prime}}-s} \psi_{l^{\prime}, k^{\prime}}\right\|_{0}\left\|\Lambda^{\bar{m}_{\lambda}+s} \psi_{l, k}\right\|_{0} \\
& \lesssim 2^{l \bar{m}_{\lambda}+l^{\prime} \bar{m}_{\lambda^{\prime}} 2^{-s\left|l-l^{\prime}\right|} .}
\end{aligned}
$$

We treat the dual wavelets in $H^{-m(x)}(I)$ analogously.

Lemma 8 In case $\operatorname{supp} \widetilde{\psi}_{\lambda} \cap \operatorname{supp} \widetilde{\psi}_{\lambda^{\prime}} \neq \emptyset$ and $l \leq l^{\prime}$, the following estimate holds for $0 \leq s+\bar{m}_{\lambda}<\gamma$,

$$
\left|\left\langle\Lambda^{-m} \widetilde{\psi}_{l, k}, \Lambda^{-m} \widetilde{\psi}_{l^{\prime}, k^{\prime}}\right\rangle\right| \lesssim 2^{-\left|l-l^{\prime}\right| s} 2^{-l \underline{m}_{\lambda}-l^{\prime} \underline{m}_{\lambda^{\prime}}} .
$$

We remark that Lemmas 6-8 include the boundary wavelets as well.

Lemma 9 For $\lambda=(l, k) \in \mathcal{I}$ one has

$$
\bar{m}_{\lambda}-\underline{m}_{\lambda} \lesssim 2^{-l}
$$

Proof Due to $m \in C^{1}(\mathbb{R})$ (actually, only $m \in C^{0,1}(\mathbb{R})$ is required) there holds

$$
0 \leq \bar{m}_{\lambda}-\underline{m}_{\lambda} \leq \operatorname{diam}\left(\Omega_{\lambda}\right) \sup _{x \in \Omega_{\lambda}}\left|m^{\prime}(x)\right| \lesssim 2^{-l} \sup _{x \in \Omega_{\lambda}}\left|m^{\prime}(x)\right| .
$$


Definition 2 A matrix $\mathbf{A}=\left(a_{\lambda, \lambda^{\prime}}\right)_{\lambda, \lambda^{\prime} \in \mathcal{I}}$ will be called compressible, if there exist constants $a, b>0$ such that for all $\lambda=(l, k), \lambda^{\prime}=\left(l^{\prime}, k^{\prime}\right)$ holds

$$
\left|a_{\lambda, \lambda^{\prime}}\right| \lesssim 2^{-(a+1 / 2)\left|l-l^{\prime}\right|}\left(1+d\left(\lambda, \lambda^{\prime}\right)\right)^{-1-b}
$$

with $d\left(\lambda, \lambda^{\prime}\right):=2^{\min \left\{\ell, \ell^{\prime}\right\}} \operatorname{dist}\left(\operatorname{supp} \psi_{\lambda}\right.$, supp $\left.\psi_{\lambda^{\prime}}\right)$ if at least one wavelet has vanishing moments of order $d^{*} \geq 2$ and $d\left(\lambda, \lambda^{\prime}\right):=+1$ otherwise.

Let us introduce the diagonal matrices

$$
\mathbf{D}^{-m(x)}:=\left(2^{-\bar{m}_{\lambda}} \delta_{\lambda, \lambda^{\prime}}\right)_{\lambda, \lambda^{\prime} \in \mathcal{I}}, \quad \mathbf{D}^{m(x)}:=\left(2^{\underline{m}_{\lambda}} \delta_{\lambda, \lambda^{\prime}}\right)_{\lambda, \lambda^{\prime} \in \mathcal{I}}
$$

Proposition 2 The matrices

$$
\begin{aligned}
\mathbf{A}=\left(a_{\lambda, \lambda^{\prime}}\right)_{\lambda, \lambda^{\prime} \in \mathcal{I}} & :=\mathbf{D}^{-m(x)} \mathbf{M D}^{-m(x)} \text { and } \\
\mathbf{A}^{(-1)} & :=\mathbf{D}^{m(x)} \mathbf{M}^{(-1)} \mathbf{D}^{m(x)}
\end{aligned}
$$

with $\mathbf{M}, \mathbf{M}^{-1}$ as in (5.1), (5.2) are compressible: there exists $s>0$ such that

$$
\left|a_{\lambda, \lambda^{\prime}}\right| \lesssim 2^{-\left|l-l^{\prime}\right|(s+1 / 2)}\left(1+d\left(\lambda, \lambda^{\prime}\right)\right)^{-1-2(d-\bar{m})(1-\delta)}
$$

and

$$
\left|a_{\lambda, \lambda^{\prime}}^{(-1)}\right| \lesssim 2^{-\left|l-l^{\prime}\right|(s+1 / 2)}\left(1+d\left(\lambda, \lambda^{\prime}\right)\right)^{-1-2(d-\underline{m})(1-\delta)} .
$$

Proof If $d\left(\lambda, \lambda^{\prime}\right) \lesssim 1$ the assertion follows from Lemma 7 and Lemma 8 .

If $d\left(\lambda, \lambda^{\prime}\right) \geq 1$ we infer from Lemma 4 , that for $l \leq l^{\prime}$ holds

$$
\begin{aligned}
\left|a_{\lambda, \lambda^{\prime}}\right| & \lesssim 2^{l(1 / 2+\bar{m}+2(1-\delta) d)-d)} 2^{-l^{\prime}(1 / 2+d-\bar{m})}\left(1+d\left(\lambda, \lambda^{\prime}\right)\right)^{-(1+2 \bar{m}+(1-\delta) 2 d)} \\
& \lesssim 2^{\left(l-l^{\prime}\right)(1 / 2+s)}\left(1+d\left(\lambda, \lambda^{\prime}\right)\right)^{-(1+2 \bar{m}+(1-\delta) 2 d))}
\end{aligned}
$$

provided that $0<s=\min \{\bar{m}+2(1-\delta) d-d, d-\bar{m}\}$, which is possible since $\delta>0$ can be arbitrarily small and $\bar{m}<1$ if $X_{t}$ is quadratic, pure jump.

An estimate for $\mathbf{A}^{(-1)}$ can be derived similarly. The case that the dual wavelets are boundary wavelets is treated similarly if only one of the wavelets has vanishing moments.

The case that neither of the wavelets has vanishing moments is treated in the same way as the case of overlapping support.

The previous result is the basis for the numerical analysis of variable order pseudodifferential equations. It implies multilevel norm equivalences in the variable order Sobolev spaces $\tilde{H}^{m(x)}(I)$ and diagonal preconditioning for wavelet discretizations of the variable order pseudo-differential operators $\Lambda^{m(x)}$.

Theorem 3 Let $u=\sum_{\lambda \in \mathcal{I}} u_{\lambda} \psi_{\lambda}=\mathbf{u}^{\top} \Psi \in \widetilde{H}^{m(x)}(I)$. Then for $\mathbf{D}^{m(x)}$ as in (5.12) holds the multilevel norm equivalence in variable order spaces:

$$
\|u\|_{\tilde{H}^{m(x)}(I)}^{2} \sim \mathbf{u}^{\top}\left(\mathbf{D}^{m(x)}\right)^{\top} \mathbf{D}^{m(x)} \mathbf{u} \sim \mathbf{u}^{\top} \mathbf{D}^{2 m(x)} \mathbf{u} .
$$


For $v=\sum_{\lambda \in \mathcal{I}} v_{\lambda} \widetilde{\psi}_{\lambda}=\mathbf{v}^{\top} \widetilde{\Psi} \in H^{-m(x)}(I)$ holds correspondingly

$$
\|v\|_{H^{-m(x)}(I)}^{2} \sim \mathbf{v}^{\top} \mathbf{D}^{-2 m(x)} \mathbf{v} .
$$

The spectral condition number of $\mathbf{A}=\mathbf{D}^{-m(x)} \mathbf{M D}^{-m(x)}$ is uniformly bounded in $h$.

Proof As a first step, we need to show that cond $\mathbf{D}^{-m(x)} \mathbf{D}^{m(x)} \leq C$. Recalling the definition we observe that $\mathbf{D}^{-m(x)} \mathbf{D}^{m(x)}=\operatorname{diag}\left(2^{-l \underline{m}_{\lambda}} 2^{l \bar{m}_{\lambda}}\right)$. Due to Lemma 9 we get $\lim _{l \rightarrow \infty} 2^{l\left|\underline{m}_{\lambda}-\bar{m}_{\lambda}\right|}=\lim _{l \rightarrow \infty} 2^{l C\left(2^{-l}\right)}=1$. Therefore we estimate $1 \leq 2^{l\left|\underline{m}_{\lambda}-\bar{m}_{\lambda}\right|} \leq C$. It is well known that the spectral norm of a compressible matrix is bounded, see e.g. [5] Theorem 4.6.1. Therefore, it follows from this result together with Proposition 2 that the spectral norm of the matrices $\mathbf{A}$ and $\mathbf{A}^{-1}$ are bounded, which means cond $\mathbf{A} \sim 1$. Thus the matrices $\mathbf{M}, \mathbf{D}^{m(x)}\left(\mathbf{D}^{m(x)}\right)^{\top}$ and $\mathbf{D}^{2 m(x)}$ are spectrally equivalent. Since $\|u\|_{\tilde{H}^{m(x)}(I)}^{2}=\mathbf{u}^{\top} \mathbf{M u}$, we have established the first assertion (5.15). The second equivalence (5.16) follows analogously.

An immediate consequence of the norm-equivalence (5.15) is the uniform boundedness of the quasi-interpolants $Q_{h}$ in the variable order norms $\tilde{H}^{m(x)}(I)$.

Corollary 1 The quasi-interpolants $Q_{h}$ defined in (4.9) are uniformly bounded in $\tilde{H}^{m(x)}(I)$, i.e. there is $C>0$ such that

$$
\forall u \in \tilde{H}^{m(x)}(I), \forall h>0: \quad\left\|Q_{h} u\right\|_{\tilde{H}^{m(x)}(I)} \leq C\|u\|_{\tilde{H}^{m(x)}(I)} .
$$

Using the variable order norm equivalence (5.15) we can extend the approximation property (4.10) to variable order spaces.

Corollary 2 Let $u \in H^{s}(I) \cap \tilde{H}^{1}(I)$ for some $1 \leq s \leq p+1$. Then for the quasiinterpolant $u_{h}=Q_{h} u=\sum_{l=0}^{L} \sum_{j=1}^{M^{l}} u_{j}^{l} \psi_{j}^{l}$ in (4.9) there holds for $0<\bar{m}<1 \leq s \leq$ $p+1$ the Jackson estimate

$$
\begin{aligned}
\left\|u-u_{h}\right\|_{\tilde{H}^{m(x)}(I)}^{2} & \lesssim \int_{I} 2^{2 L(m(x)-s)}\left(\left|D^{s} u(x)\right|^{2}+|u(x)|^{2}\right) d x \\
& \lesssim 2^{2 L(\bar{m}-s)}\|u\|_{H^{s}(I)}^{2} .
\end{aligned}
$$

Proof For multi-indices $\lambda=(l, k), \mu=\left(L, k^{\prime}\right) \in \mathcal{I}$, we introduce the notation $\lambda \succeq \mu$ if $l:=|\lambda| \geq|\mu|=: L$ and $\operatorname{supp} \psi_{\lambda} \cap \operatorname{supp} \psi_{\mu} \neq \emptyset$. For $s \geq \frac{3}{2}$ we choose $s^{\prime}<s$ with $1 \leq s^{\prime}<\frac{3}{2}$, otherwise we set $s^{\prime}=s$, we observe that $\underline{m}_{\lambda}-s^{\prime} \leq\left(\bar{m}_{\mu}-s^{\prime}\right)<0$ holds for all $\lambda \succeq \mu$. Therefore we conclude from the norm equivalence (5.15)

$$
\begin{aligned}
\left\|u-u_{h}\right\|_{\tilde{H}^{m(x)}(I)}^{2} & \sim \sum_{l>L} \sum_{k=1}^{M_{l}} 2^{2|\lambda| \underline{m}_{\lambda}}\left|u_{l, k}\right|^{2} \\
& =\sum_{l>L} \sum_{k=1}^{M_{l}} 2^{2|\lambda|\left(\underline{m}_{\lambda}-s^{\prime}\right)} 2^{2 s^{\prime}|\lambda|}\left|u_{\lambda}\right|^{2}
\end{aligned}
$$




$$
\lesssim \sum_{\mu \in \nabla_{L}} 2^{2 L\left(\bar{m}_{\mu}-s^{\prime}\right)} \sum_{\lambda \succeq \mu} 2^{2 s^{\prime}|\lambda|}\left|u_{\lambda}\right|^{2}
$$

where $\nabla_{L}=\left\{\mu=\left(L, k^{\prime}\right): k^{\prime}=1, \ldots, M_{L}\right\}$.

Let $\mu=\left(L, k^{\prime}\right), L=|\mu|$ and $\square_{\mu}:=\left[2^{-|\mu|} k^{\prime}, 2^{-|\mu|}\left(k^{\prime}+1\right)\right]$. Then, due to the norm equivalence (5.15) and the approximation property (4.10) we have

$$
\sum_{\mu \in \nabla_{L}} \sum_{\lambda \succeq \mu} 2^{2 s^{\prime}|\lambda|}\left|u_{\lambda}\right|^{2} \lesssim \sum_{\mu \in \nabla_{L}} 2^{2 L\left(s^{\prime}-s\right)} \int_{\square_{\mu}}\left(\left|D^{s} u(x)\right|^{2}+|u(x)|^{2}\right) d x .
$$

Recalling that $2^{L \underline{m}} \mu \sim 2^{L m(x)} \sim 2^{L \bar{m}_{\mu}}$ holds for $x \in \square_{\mu}$, we obtain the final result

$$
\left\|u-u_{h}\right\|_{\tilde{H}^{m(x)}(I)}^{2} \lesssim \int_{I} 2^{2 L(m(x)-s)}\left(\left|D^{s} u(x)\right|^{2}+|u(x)|^{2}\right) d x
$$

Lemmas 4 to 8 addressed only the generators $\Lambda^{m(x)}$. We focused on this case since it is crucial for establishing the norm equivalences (5.15). For wavelet solution of the Kolmogoroff equation (1.8) in log-linear complexity we need the compressibility of the moment matrices in wavelet bases also for generators which are more general than $\Lambda^{m(x)}$.

Theorem 4 Let $A \in \Psi_{\rho, \delta}^{2 m(x)}(\mathbb{R})$ for some $0<\delta<\rho \leq 1$, and let $\left\{\psi_{\lambda}\right\}_{\lambda}$ be a system of compactly supported spline wavelets in $I=(0,1)$ of polynomial degree $p \geq 1$ as introduced in Sect. 4 with complementary boundary conditions and $d \geq p+1$ vanishing moments if $\operatorname{supp}\left(\psi_{\lambda}\right) \cap \partial I=\emptyset$.

Then the preconditioned moment matrix

$$
\mathbf{A}:=\mathbf{D}^{-m(x)}\left(\left\langle A \psi_{\lambda^{\prime}}, \psi_{\lambda}\right\rangle\right)_{\lambda, \lambda^{\prime} \in \mathcal{I}} \mathbf{D}^{-m(x)}
$$

has condition number which is uniformly bounded in $L$ and $\mathbf{A}$ is compressible: there are $C>0, s>0$ such that for all $\lambda, \lambda^{\prime} \in \mathcal{I}$ holds

$$
\left|\mathbf{A}_{\lambda, \lambda^{\prime}}\right| \leq C 2^{-\left|l-l^{\prime}\right|(s+1 / 2)}\left(1+d\left(\lambda, \lambda^{\prime}\right)\right)^{-1-2(d-\bar{m})(\rho-\delta)} .
$$

The proof of Theorem 4 is completely analogous to that of Proposition 2, using Lemmas 4 to 8 with the kernel estimates (2.15) in place of (2.7).

\section{Option pricing under variable intensity feller processes}

With the norm equivalences (5.15), (5.16) we develop linear complexity numerical solution algorithms for the pricing equation (1.8). Since elements of the algorithm are identical to $[22,23,26]$, we focus only on the essential differences in the analysis due to the variable order $m(x)$ of $A_{X}$. 
6.1 Weak form of the pricing equation. Well-posedness

We consider (1.8) with nonlocal operator $A \in \mathcal{L}\left(V, V^{*}\right)$ of variable order $2 m(x) \in$ $(0,2)$ in $V=\widetilde{H}^{m(x)}(I), V^{*}=H^{-m(x)}(I)$ with Dirichlet form

$$
a(u, v):=\langle A u, v\rangle_{V^{*} \times V}, \quad u, v \in V .
$$

This Dirichlet form is continuous, i.e. there exists a constant $\alpha>0$ such that

$$
|a(u, v)| \leq \alpha\|u\|_{V}\|v\|_{V}, \quad \forall u, v \in V .
$$

It is well known that problem (1.8) has a unique (weak) solution $u(t)$, and there holds the a priori estimate (see e.g. [19])

$$
\|u\|_{\mathcal{C}\left(\bar{J}, L^{2}(I)\right)}+\|u\|_{L^{2}(J, V)}+\|\dot{u}\|_{L^{2}\left(J, V^{*}\right)} \leq C\left\|u_{0}\right\|_{L^{2}(I)}
$$

provided that for some constants $\gamma>0, C \geq 0$ the Dirichlet form $a(\cdot, \cdot)$ satisfies the Gårding inequality, i.e.,

$$
\forall u \in V \quad \Re a(u, u) \geq \gamma\|u\|_{V}^{2}-C\|u\|_{L^{2}(I)}^{2} .
$$

Remark 1 Without loss of generality, we may assume in (6.2) that $C=0$ since the substitution $\bar{u}=e^{-\beta t} u$ results in (1.8) with the shifted operator $A+\beta I$ which is definite for sufficiently large $\beta>0$ by (6.2) $(\beta>2 C$ will suffice).

Note that for asymmetric distributions of positive and negative jumps in $X_{t}$, the generator $A$ needs not to be self-adjoint, i.e. $a(\cdot, \cdot)$ is generally non-symmetric.

\subsection{Gårding inequality. Analyticity of $T_{t}$}

To establish (6.2), we start with the variable order Riesz potential $A=\Lambda^{2 m(x)} \in$ $\Psi_{1, \delta}^{2 m(x)}$ given by

$$
A u(x):=\frac{1}{2 \pi} \iint e^{i \xi \cdot(x-y)}\langle\xi\rangle^{2 m(x)} u(y) d y d \xi, \quad u \in C_{0}^{\infty}(I),
$$

and introduce the operator $L=\Lambda^{m(x)} \in \Psi_{1, \delta}^{m(x)}$.

Lemma 10 For every $\delta>0$ sufficiently small there exist $\varepsilon>0$ and pseudodifferential operators $B, R \in \Psi_{1, \delta}^{-\varepsilon}$ with symbols $b(x, \xi), r(x, \xi) \in S_{1, \delta}^{-\varepsilon}$ such that

$$
A=L \circ L^{\top}+L \circ B+R
$$

Proof Since the extended symbol $a(x, \xi, y)$ of $L L^{\top}$ is in $S_{1, \delta}^{2 \bar{m}}$, and since $I \subset \mathbb{R}$ is compact we can assume that $A(x, D, y)$ is compactly supported. We also may apply standard pseudo differential operator calculus and expand 


$$
\begin{aligned}
a(x, \xi, y) & =a_{0}(x, \xi)+a_{1}(x, \xi)+r_{1}(x, \xi, y) \\
& =\langle\xi\rangle^{2 m(x)}+\left.D_{y} D_{\xi}(\langle\xi\rangle)^{m(x)+m(y)}\right|_{y=x}+r_{1}(x, \xi, y) .
\end{aligned}
$$

Therefore, the subprincipal symbol of $a(x, \xi)$ is

$$
a_{1}(x, \xi)=\langle\xi\rangle^{m(x)}\langle\xi\rangle^{m(x)-1} \log \langle\xi\rangle .
$$

We represent the corresponding operator $a_{1}(x, D)$, using symbolic calculus, as

$$
a_{1}(x, D)=L \circ\langle D\rangle^{m(x)-1} \log \langle D\rangle+R_{2}=L \circ B+R_{2},
$$

where $R_{2} \in \Psi_{1, \delta}^{-\varepsilon}$ for sufficiently small $\varepsilon$. The symbol of the operator $B$ is $b(x, \xi)=$ $\langle\xi\rangle^{m(x)-1} \log \langle\xi\rangle \in S_{I, \delta}^{-\varepsilon}$, provided that $0<\varepsilon<1-\delta-\bar{m}$. Setting $R=R_{1}+R_{2}$ gives the claimed result.

Lemma 11 Let $B \in \Psi_{1, \delta}^{-\varepsilon}$ and $b \in S_{1, \delta}^{-\varepsilon}$ as in Lemma 10. Then, for each $\epsilon>0$ there exists $b_{\epsilon} \in S_{1, \delta}^{-2}$ such that

$$
\left|\left(b-b_{\epsilon}\right)(x, \xi)\right| \leq \epsilon .
$$

Proof Since $b \in S_{1, \delta}^{-\varepsilon}$, for any $\epsilon>0$ there exists $R_{\epsilon}$ such that

$$
|b(x, \xi)| \leq \epsilon, \quad|\xi| \geq R_{\epsilon}
$$

holds. Let $\chi$ be the cut-off function defined in the proof of Lemma 2, we set $\hat{\chi}_{R_{\epsilon}}(\xi):=$ $\hat{\chi}\left((2 R)_{\epsilon}^{-1}\left(\xi-\frac{1}{2}\right)\right)$ and $b_{\epsilon}(x, \xi):=b(x, \xi) \chi_{R_{\epsilon}}(\xi)$. Then

$$
b_{\epsilon}(x, \xi)=0, \quad x \in \mathbb{R},|\xi|>2 R_{\epsilon}
$$

i.e. $b_{\epsilon} \in S_{1, \delta}^{-k}$ for all $k \in \mathbb{N}$ and $b_{\epsilon}$ satisfies the desired estimates (6.5).

Lemma 12 Let the operators $B$ be defined as in Lemma 10 , and $B_{\epsilon}$ be defined by the symbol $b_{\epsilon}$ in Lemma 11. Then there exists a constant $C>0$ s.t.

$$
\left|\left\langle L \circ\left(B-B_{\epsilon}\right) u, v\right\rangle\right| \leq C \epsilon\|u\|_{\tilde{H}^{m(x)}(I)}\|v\|_{\tilde{H}^{m(x)}(I)}
$$

and

$$
\left|\left\langle L \circ B_{\epsilon} u, u\right\rangle\right| \leq C \epsilon\|u\|_{L_{2}(I)}^{2}
$$

hold for all $\epsilon>0$ and for all $u, v \in \widetilde{H}^{m(x)}(I)$.

Proof Since $u, v$ have compact support, we can assume without loss of generality that $x \mapsto b(x, \xi)-b_{\epsilon}(x, \xi)$ has compact support with respect to $x$, which simplifies the proof. We proceed in a standard way (see e.g. [34], Chap. VI, §2), by proving that under this assumption Lemma 11 ensures that for all $\epsilon>0$ there exists $B_{\epsilon}$ satisfying

$$
\left\|\left(B-B_{\epsilon}\right) u\right\|_{L_{2}(\mathbb{R})} \leq \epsilon\|u\|_{L_{2}(I)} .
$$


To this end, let us define the function $\hat{d}_{\epsilon}(\lambda, \xi):=\frac{1}{2 \pi} \int e^{-i x \lambda}\left(b(x, \xi)-b_{\epsilon}(x, \xi)\right) d x$. Due to the local support of $x \mapsto b(x, \xi)-b_{\epsilon}(x, \xi)$, for each $N \in \mathbb{N}, \epsilon>0$, there holds the estimate

$$
\sup _{\xi \in \mathbb{R}}\left|\hat{d}_{\epsilon}(\lambda, \xi)\right| \leq C_{N} \epsilon(1+|\lambda|)^{-N}
$$

We write

$$
\begin{aligned}
\left(B-B_{\epsilon}\right) u(x) & =\frac{1}{2 \pi} \iint e^{i \xi(x-y)}\left(b(x, \xi)-b_{\epsilon}(x, \xi)\right) u(y) d y d \xi \\
& =\frac{1}{2 \pi} \iint\left(\int \hat{d}_{\epsilon}(\lambda, \xi) e^{i \lambda x} d \lambda\right) e^{i \xi(x-y)} u(y) d y d \xi \\
& =\int\left(e^{i x \lambda} \int\left(e^{i x \xi} \hat{d}_{\epsilon}(\lambda, \xi) \hat{u}(\xi) d \xi\right)\right) d \lambda=: \int\left(D_{\lambda}^{\epsilon} u\right)(x) d \lambda
\end{aligned}
$$

By Plancherel's Theorem we get for $N=2$ in (6.8)

$$
\left\|D_{\lambda} u\right\|_{L_{2}(\mathbb{R})} \lesssim \sup _{\xi \in \mathbb{R}}\left|\hat{d}_{\epsilon}(\lambda, \xi)\right|\|\hat{u}\|_{L_{2}(\mathbb{R})} \lesssim \epsilon(1+|\lambda|)^{-2}\|u\|_{L_{2}(\mathbb{R})} .
$$

Integrating with respect to $\lambda$ gives, for every $u \in L^{2}(\mathbb{R})$

$$
\begin{aligned}
\left\|\left(B-B_{\epsilon}\right) u\right\|_{L_{2}(\mathbb{R})} & \lesssim \int_{\lambda \in \mathbb{R}}\left\|D_{\lambda}^{\epsilon} u\right\|_{L_{2}(\mathbb{R})} d \lambda \\
& \lesssim \int_{\lambda \in \mathbb{R}}\left((1+|\lambda|)^{-2}\right) \epsilon\|u\|_{L_{2}(\mathbb{R})} d \lambda \lesssim \epsilon\|u\|_{L_{2}(\mathbb{R})}
\end{aligned}
$$

Therefore, we estimate

$$
\begin{aligned}
\left|\left\langle L \circ\left(B-B_{\epsilon}\right) u, v\right\rangle\right| & \leq\left\|\left(B-B_{\epsilon}\right) u\right\|_{L_{2}(\mathbb{R})}\left\|\Lambda^{m(x)} v\right\|_{L_{2}(\mathbb{R})} \\
& \leq \epsilon\|u\|_{L_{2}(I)}\|v\|_{\tilde{H}^{m(x)}(I)} \\
& \leq \epsilon\|v\|_{\tilde{H}^{m(x)}(I)}\|u\|_{\tilde{H}^{m(x)}(I)},
\end{aligned}
$$

for all $u, v \in \widetilde{H}^{m}(I)$, which concludes the first estimate (6.6).

Since $\bar{m}<1$ and $b_{\epsilon} \in S_{1, \delta}^{-2}$, standard pseudo differential operator calculus gives $L \circ B_{\epsilon} \in \Psi_{1, \delta}^{-\varepsilon}$ and we obtain

$$
\left|\left\langle L \circ B_{\epsilon} u, u\right\rangle\right| \lesssim\|u\|_{L_{2}(I)}^{2}
$$

Now we establish the Gårding inequality in an important special case. We recall the definition (3.3) of the $\tilde{H}^{m(x)}(I)$-norm. 
Lemma 13 The variable order Riesz potential operator A given by (6.3) satisfies the Gårding inequality: there exist constants $\gamma>0$ and $C \geq 0$ such that $\forall u \in \tilde{H}^{m(x)}(I)$ :

$$
\Re a(u, u)=\frac{1}{2}(\langle A u, u\rangle+\langle u, A u\rangle) \geq \gamma\|u\|_{\tilde{H}^{m(x)}(I)}^{2}-C\|u\|_{L_{2}(I)}^{2} .
$$

Furthermore, the operator A is continuous,

$$
|\langle A u, v\rangle| \lesssim\|u\|_{\tilde{H}^{m(x)}(I)}\|v\|_{\tilde{H}^{m(x)}(I)} \quad \forall u, v \in \widetilde{H}^{m(x)}(I) .
$$

Proof We decompose

$$
A=L \circ L^{\top}+L \circ\left(B-B_{\epsilon}\right)+L \circ B_{\epsilon}+R
$$

where $R \in \Psi_{1, \delta}^{-\varepsilon}$. A similar expression holds for the adjoint $A^{\top}$. Using Lemma 12 there exist constants $\gamma>0, C^{\prime} \geq 0$ such that for $\epsilon>0$ sufficiently small and $\forall u \in$ $\tilde{H}^{m(x)}(I)$ :

$$
\begin{aligned}
\langle A u, u\rangle+\langle u, A u\rangle & \geq 2\left\langle\Lambda^{m(x)} u, \Lambda^{m(x)} u\right\rangle-\epsilon\|u\|_{\tilde{H}^{m(x)}(I)}^{2}-C\|u\|_{L_{2}(I)}^{2} \\
& \geq \gamma\|u\|_{\tilde{H}^{m(x)}(I)}^{2}-C^{\prime}\|u\|_{L_{2}(I)}^{2},
\end{aligned}
$$

which proves the Gårding inequality (6.9).

The second assertion follows by the same ingredients observing the $L_{2}$-continuity of $R$ and $B_{\epsilon}$.

The previous theorem has been shown in [12] for a larger class of operators under the more restrictive condition $\bar{m}-\underline{m}<\frac{1}{2}$.

The Garding inequality (6.9) holds for the operator $A=\Lambda^{2 m(x)}$ defined in (6.3). Lemma 13 is, however, the key ingredient in establishing a Gårding inequality for variable order operators $A(x, D) \in \Psi_{\rho, \delta}^{2 m(x)}$ which are elliptic in a suitable sense.

Theorem 5 Let $A(x, D) \in \Psi_{\rho, \delta}^{2 m(x)}$ be a pseudodifferential operator of variable order $2 m(x), 0<m(x)<1$, given by (2.3) with symbol $a(x, \xi) \in S_{\rho, \delta}^{2 m(x)}$ for some $0<\delta<$ $\rho \leq 1$ for which there exists $\gamma>0$ with

$$
\forall x, \xi \in \mathbb{R}^{d}: \quad \text { Re } a(x, \xi)+1 \geq \gamma\langle\xi\rangle^{2 m(x)} .
$$

Then $A(x, D) \in \Psi_{\rho, \delta}^{2 m(x)}$ satisfies a Gårding inequality in the variable order space $\tilde{H}^{m(x)}(I)$ : there are constants $\gamma>0$ and $C \geq 0$ such that

$$
\forall u \in \tilde{H}^{m(x)}(I): \quad \Re a(u, u) \geq \gamma\|u\|_{\tilde{H}^{m(x)}(I)}^{2}-C\|u\|_{L_{2}(I)}^{2},
$$

and

$$
\exists \lambda>0 \quad \text { such that } A(x, D)+\lambda I: \tilde{H}^{m(x)}(I) \rightarrow H^{-m(x)}(I)
$$

is boundedly invertible. 
Proof Let $I=(a, b) \subset \mathbb{R}$ be a bounded interval and $m(x): I \rightarrow(0,1)$ a variable order. As in the proof of Lemma 13 , we can decompose $A$ into

$$
A=N \circ N^{\top}+N\left(B-B_{\epsilon}\right)+N \circ B_{\epsilon}+R,
$$

where $N$ is an injective elliptic operator on $I$ with symbol $n(x, \xi)$ satisfying $n(x, \xi) \geq$ $c\langle\xi\rangle^{m(x)}$ and $B, B_{\epsilon}$ and $R$ have similar properties as in Lemmas 10-12. Due to these assumptions there is $c>0$ such that for all $u \in \tilde{H}^{m(x)}(I)$ we have $\|N u\|_{L^{2}(I)} \geq$ $c\|u\|_{\tilde{H}^{m(x)}(I)}$. This implies in the same fashion as in the previous proof that there exist constants $c, C^{\prime}, \gamma^{\prime}>0$ s.t. for all $u \in \tilde{H}^{m(x)}(I)$ :

$$
\begin{aligned}
\Re a(u, u) & =\frac{1}{2}(\langle A u, u\rangle+\langle u, A u\rangle)=c \gamma\left((N u, N u)-\|u\|_{L^{2}(I)}^{2}\right) \\
& \geq \gamma^{\prime}\left(\|u\|_{\tilde{H}^{m(x)}(I)}^{2}-C^{\prime}\|u\|_{L^{2}(I)}^{2}\right) .
\end{aligned}
$$

Where we conclude (6.9) with Lemmas 10 to 13.

The bounded invertibility (6.13) of $A(x, D)+\lambda I$ follows from (6.12): it implies that for $\lambda>0$ sufficiently large, $A(x, D)+\lambda I$ is $\tilde{H}^{m(x)}(I)$-coercive.

The Gårding inequality (6.12) implies time-analyticity of the semigroup $T_{t}$ corresponding to $X \in F P_{\rho, \delta}^{2 m(x)}$ and $A \in \Psi_{\rho, \delta}^{2 m(x)}$. As in [32], Theorem 1, we obtain from (6.9)

Theorem 6 Let $X \in F P_{\rho, \delta}^{2 m(x)}$ be a quadratic, pure jump Feller process of variable intensity $2 m(x)$ with characteristic function $a(x, \xi)$ as in (1.6) belonging to $S_{\rho, \delta}^{2 m(x)}$, which we assume to be coercive in the sense that (6.11) holds. Then $A(x, D)$ is the infinitesimal generator of the Feller semigroup $T_{t}$ of $X_{t}$ defined in (1.2). This semigroup is analytic in the sense, that there exist $C, d>0$, such that for all $t>0, l \in \mathbb{N}_{0}$ and $0 \leq \theta \leq 1$ holds

$$
\left\|T^{(l)}(t)\right\|_{\mathcal{L}\left(V_{\theta}, V\right)}^{2} \leq C d^{2 l+1-2 \theta} \Gamma(2 l+2-2 \theta) t^{-2(l+1)+2 \theta} .
$$

Here, $V_{\theta}=\left(H^{-m(x)}(I), \tilde{H}^{m(x)}(I)\right)_{\theta, 2}$ denotes the real interpolation space with index $0 \leq \theta \leq 1$ with the convention that $V=V_{1}=\tilde{H}^{m(x)}(I)$.

Proof If the negative definite characteristic function $a(x, \xi)$ in (1.6) belongs to $S_{\rho, \delta}^{2 m(x)}$, by Theorem 1.3 of [30], the operator $A(x, D)$ extends to a Feller generator given by (1.3). Assumption (6.11) implies by Theorem 5 and by Theorem 1 in [32] the analytic regularity estimate (6.14) for the Feller semigroup $T_{t}$.

\subsection{Discretization of the Kolmogoroff equation}

We discretize (1.8) with wavelets on $I=(a, b)$, and, to exploit the time-analyticity (6.14) of $u(t)$, by a $h p$-discontinuous Galerkin (dG) scheme on $J=(0, T)$, following 
$[22,26,32]$. In our analysis, we will need to consider functions in $V=\widetilde{H}^{m(x)}(I)$ with additional regularity. Therefore, for $s \geq 0$, we define the spaces

$$
\mathcal{H}^{s}(I)= \begin{cases}\widetilde{H}^{s}(I) & \text { for } s \leq \bar{m}, \\ V \cap H^{s}(I) & \text { for } s>\bar{m} .\end{cases}
$$

By $\|\cdot\|_{s}$, we denote the corresponding norm in $\mathcal{H}^{s}(I)$.

\subsubsection{Spatial semi-discretization by wavelets}

We first discretize (1.8) with respect to the space variable. Hence, let $\mathcal{T}^{0}$ be a fixed coarse partition of $I$. Furthermore, define the mesh $\mathcal{T}^{l}$, for $l>0$, recursively by bisection of each interval in $\mathcal{T}^{l-1}$. We assume that our computational mesh $\mathcal{T}_{h}$ (with mesh size $h$ ) is obtained in this way as $\mathcal{T}^{L}$, for some $L>0$, with $h=C 2^{-L}$.

The finite element space $V_{h} \subset V$ used for the discretization is the space of all continuous piecewise polynomials of degree $p \geq 1$ on the triangulation $\mathcal{T}_{h}$ which vanish on the boundary $\partial I$. In the same way, we define the spaces $V^{l}$ corresponding to the triangulation $\mathcal{T}^{l}$, so that we have $V^{0} \subset V^{1} \subset \cdots \subset V^{L}=V_{h}$. Let $N^{l}=\operatorname{dim} V^{l}$ and $N=\operatorname{dim} V_{h}=N^{L}=C 2^{L}$.

The semi-discrete problem corresponding to (1.8) reads: Given $u_{0} \in L^{2}(I)$, find $u_{h} \in H^{1}\left(J, V_{h}\right)$ such that

$$
\frac{d}{d t}\left(u_{h}, v_{h}\right)+a\left(u_{h}, v_{h}\right)=0, \quad \forall v_{h} \in V_{h}, \text { and } u_{h}(0)=P_{h} u_{0}
$$

Here, $P_{h}$ is the $L^{2}$ projection onto $V_{h}$. Using the stability of the quasi interpolant $Q_{h}$ in (4.9), i.e. Corollary 1 , the following result on the spatial semi-discretization can be proved as in [23], Sect. 5, using the approximation property Corollary 2.

Theorem 7 Let (6.1) and (6.9) be satisfied. Furthermore, we assume that the operator $A$ is of the form $A=A_{0}+\mathcal{B}$, where $A_{0}=C_{0} \Lambda^{2 m(x)} \in \Psi_{1, \delta}^{2 m(x)}, C_{0}>0$ and $\delta>0$ arbitrarily small, is a variable order Riesz potential, and $\mathcal{B}: V \rightarrow V^{*}$ is a compact perturbation in $V=\tilde{H}^{m(x)}(I)$.

Then, for any $t>0$, there holds

$$
\left\|u(t)-u_{h}(t)\right\|_{L^{2}(I)} \leq C \min \left\{1, h^{p+1} t^{-\frac{p+1}{\bar{m}}}\right\} .
$$

Here, $C>0$ is a constant independent of $h$ and $t$, and $u, u_{h}$ are solutions of (1.8) and (6.16), respectively.

In order to compute a fully discrete approximation (in space and time) to the parabolic problem (1.6), systems of linear equations have to be solved in each implicit time step (of the corresponding time discretization). To obtain an efficient solution method, we use wavelet matrix compression of the dense matrices corresponding to the operator $A$ into sparse ones and we use optimal preconditioning. The methodology is as in $[22,23]$, but in the variable order case it is based on the norm equivalence 
Theorem 3 and the approximation property Corollary 2. After compression and preconditioning, the systems may be solved in linear or log-linear complexity. Since the proofs are analogous to what was done in $[22,23]$, we only give brief outlines of the argument here.

The restriction of the Dirichlet form $a$ to $V_{h} \times V_{h}$ corresponds to a matrix $\mathbf{A}$ with entries $A_{\lambda, \lambda^{\prime}}=A_{(l, k),\left(l^{\prime}, k^{\prime}\right)}=a\left(\psi_{l^{\prime}, k^{\prime}}, \psi_{l, k}\right)$ which, due to the Caldéron-Zygmund estimates (2.15) and the vanishing moment conditions (4.4)-(4.6), decay with increasing distance of their supports. Hence, we can define a compressed matrix $\widetilde{\mathbf{A}}$ and a corresponding sesquilinear form $\widetilde{a}$ by replacing some of the small entries in $\mathbf{A}$ with zero:

$$
\widetilde{A}_{(l, k),\left(l^{\prime}, k^{\prime}\right)}= \begin{cases}A_{(l, k),\left(l^{\prime}, k^{\prime}\right)} & \text { if } \operatorname{dist}\left(\Omega_{l, k}, \Omega_{l^{\prime}, k^{\prime}}\right) \leq \delta_{l, l^{\prime}} \text { or } \Omega_{l, k} \cap \partial I \neq \emptyset, \\ 0 & \text { otherwise. }\end{cases}
$$

Here, the truncation parameters $\delta_{l, l^{\prime}}$ are given by

$$
\delta_{l, l^{\prime}}:=c \max \left\{2^{-L+\widehat{\alpha}\left(2 L-l-l^{\prime}\right)}, 2^{-l}, 2^{-l^{\prime}}\right\},
$$

with some parameters $c>0$ and $0<\widehat{\alpha} \leq 1$ independent of $L, l, l^{\prime}$ and $\Omega_{l, k}=$ $\operatorname{supp} \psi_{l, k}$.

By continuity (6.10) and coercivity (6.9) with $C^{\prime}=0$ (which can always be achieved by Remark 1) of the Dirichlet form $a$, we have the norm equivalence

$$
\|u\|_{a}=|a(u, u)|^{\frac{1}{2}} \cong\|u\|_{V} \cong\|u\|_{\tilde{H}^{m(x)}(I)} .
$$

As in [25, Proposition 3.2], we have

Proposition 3 Assume $c$ in (6.19) is sufficiently large. Then, there exists $0<\widetilde{\beta} \leq$ $\widetilde{\alpha}<\infty$ independent of $h$ such that

$$
\left|\widetilde{a}\left(u_{h}, v_{h}\right)\right| \leq \widetilde{\alpha}\left\|u_{h}\right\|_{a}\left\|v_{h}\right\|_{a},
$$

and

$$
\Re \widetilde{a}\left(u_{h}, u_{h}\right) \geq \widetilde{\beta}\left\|u_{h}\right\|_{a}^{2},
$$

for all $u_{h}, v_{h} \in V_{h}$.

Moreover, there holds [25]

Proposition 4 Assume $c$ in (6.19) is sufficiently large. Then, there exists $0<$ $\tau<1$ independent of $h$ such that for all $L>0$ the following consistency condition is satisfied:

$$
\left|a\left(u_{h}, v_{h}\right)-\widetilde{a}\left(u_{h}, v_{h}\right)\right| \leq \tau\left\|u_{h}\right\|_{a}\left\|v_{h}\right\|_{a}, \quad \forall u_{h}, v_{h} \in V_{h}
$$

In addition, if

$$
\widehat{\alpha}>\frac{2 p+2}{2 p+2+2 \inf \{x: m(x)(\rho-\delta)\}}=\frac{p+1}{p+1+\underline{m}(\rho-\delta)},
$$


where $\widehat{\alpha}$ is the constant from (6.19), then, there holds for all $u \in \mathcal{H}^{s}(I), v_{h} \in V_{h}$ :

$$
\left|a\left(Q_{h} u, v_{h}\right)-\widetilde{a}\left(Q_{h} u, v_{h}\right)\right| \leq C h^{s-\bar{m}}\|u\|_{s}\left\|v_{h}\right\|_{V},
$$

and for all $u \in \mathcal{H}^{s}(I), v \in \mathcal{H}^{s^{\prime}}(I)$ :

$$
\left|a\left(Q_{h} u, Q_{h} v\right)-\widetilde{a}\left(Q_{h} u, Q_{h} v\right)\right| \leq C h^{s+s^{\prime}-2 \bar{m}}\|u\|_{s}\|v\|_{s^{\prime}},
$$

for all $\bar{m} \leq s, s^{\prime} \leq p+1$, with $\mathcal{H}^{s}$ as in (6.15). Here, $Q_{h}$ is a projection or quasiinterpolation operator from (4.9).

The matrix compression (6.18) reduces the number of nonzero elements from $N^{2}$ in $\mathbf{A}$ to $N$ times a logarithmic term in $\widetilde{\mathbf{A}}$; see e.g. [5, 31]. More precisely, there holds:

Proposition 5 For $\widehat{\alpha}<1$ in (6.23), the number of nonzero elements in $\widetilde{\mathbf{A}}$ is $\mathcal{O}(N \log N)$. If $\widehat{\alpha}=1$, then the number of nonzero elements in $\widetilde{\mathbf{A}}$ is $\mathcal{O}\left(N(\log N)^{2}\right)$.

The matrix compression from the previous section induces, instead of (6.16), a perturbed spatial semi-discretization of (1.8): find $\tilde{u}_{h} \in V_{h}$ such that

$$
\frac{d}{d t}\left(\tilde{u}_{h}, v_{h}\right)+\widetilde{a}\left(\tilde{u}_{h}, v_{h}\right)=0, \quad \forall v_{h} \in V_{h} \text { with } \tilde{u}_{h}(0)=P_{h} u_{0} .
$$

\subsubsection{Discontinuous Galerkin time discretization}

In order to obtain a fully discrete approximation (in space and time) to the parabolic problem (1.8), we discretize (6.25) in time using a discontinuous Galerkin timestepping following [32].

For $0<T<\infty$ and $M \in \mathbb{N}$, let $\mathcal{M}=\left\{J_{m}\right\}_{m=1}^{M}$ be a partition of $J=(0, T)$ into $M$ subintervals $J_{m}=\left(t_{m-1}, t_{m}\right), m=1,2, \ldots, M$ with $0=t_{0}<t_{1}<t_{2}<\cdots<t_{M}=T$. Moreover, denote by $k_{m}=t_{m}-t_{m-1}$ the length of $J_{m}$.

For $u \in H^{1}\left(\mathcal{M}, V_{h}\right)=\left\{v \in L^{2}\left(J, V_{h}\right):\left.v\right|_{J_{m}} \in H^{1}\left(J_{m}, V_{h}\right), m=1,2, \ldots, M\right\}$, define the one-sided limits

$$
\begin{aligned}
& u_{m}^{+}=\lim _{s \rightarrow 0^{+}} u\left(t_{m}+s\right), \quad m=0,1, \ldots, M-1, \\
& u_{m}^{-}=\lim _{s \rightarrow 0^{+}} u\left(t_{m}-s\right), \quad m=1,2, \ldots, M,
\end{aligned}
$$

and the jumps

$$
\llbracket u \rrbracket_{m}=u_{m}^{+}-u_{m}^{-}, \quad m=1,2, \ldots, M-1 .
$$

In addition, to each time interval $J_{m}$, a polynomial degree (approximation order) $r_{m} \geq 0$ is associated. These numbers are stored in the degree vector $\underline{r}=\left\{r_{m}\right\}_{m=1}^{M}$. Discontinuous Galerkin time stepping yields an approximate solution in the space

$$
\mathcal{S}^{-}\left(\mathcal{M}, V_{h}\right)=\left\{u \in L^{2}\left(J, V_{h}\right):\left.u\right|_{J_{m}} \in \mathcal{P}_{r_{m}}\left(J_{m}, V_{h}\right), m=1,2, \ldots, M\right\},
$$

where $\mathcal{P}_{r_{m}}\left(J_{m}\right)$ denotes the space of polynomials of degree at most $r_{m}$ on time interval $J_{m}$. 
With these definitions, the fully discrete $\mathrm{dG}$ scheme for the solution of problem (1.8), respectively (6.25), reads as follows: find $\widetilde{U}_{h}^{d G} \in \mathcal{S}^{-}\left(\mathcal{M}, V_{h}\right)$ such that for all $W \in \mathcal{S}^{-}\left(\mathcal{M}, V_{h}\right)$

$$
\widetilde{B}_{d G}\left(\widetilde{U}_{h}^{d G}, W\right)=F_{d G}(W)
$$

where

$$
\begin{aligned}
\widetilde{B}_{d G}(U, W)= & \sum_{m=1}^{M} \int_{J_{m}}((\dot{U}, W)+\widetilde{a}(U, W)) d t \\
& +\sum_{m=1}^{M-1}\left(\llbracket U \rrbracket_{m}, W_{m}^{+}\right)+\left(U_{0}^{+}, W_{0}^{+}\right),
\end{aligned}
$$

and

$$
F_{d G}(W)=\left(P_{h} u_{0}, W_{0}^{+}\right) .
$$

We recall from [32] that the solution $\widetilde{U}_{h}^{d G} \in \mathcal{S}^{r}\left(\mathcal{M}, V_{h}\right)$ of the $\mathrm{dG}$ method (6.26) is uniquely defined and that the $\mathrm{dG}$ method (6.26) can be interpreted as a time stepping scheme of variable step size $k_{m}$ and orders $r_{m}$. Indeed, assuming that $\widetilde{U}_{h}^{d G}$ is known on the time intervals $J_{m}=\left(t_{m-1}, t_{m}\right), m=1,2, \ldots, n-1$, we may find $\widetilde{U}_{h}^{d G} \in \mathcal{P}_{r_{n}}\left(I_{n}, V_{h}\right), 1 \leq n \leq M$, by solving the variational problem

$$
\int_{I_{n}}\left(\left(\partial_{t} \widetilde{U}_{h}^{d G}, W\right)+\widetilde{a}\left(\widetilde{U}_{h}^{d G}, W\right)\right) d t+\left(\widetilde{U}_{n-1}^{d G+}, W_{n-1}^{+}\right)=\left(\widetilde{U}_{n-1}^{d G-}, W_{n-1}^{+}\right),
$$

for all $W \in \mathcal{P}_{r_{n}}\left(I_{n}, V_{h}\right)$. Here, we set $\widetilde{U}_{0}^{d G-}=P_{h} u_{0}$.

\subsubsection{Convergence of the fully discrete scheme}

The solution operator of the parabolic problem (1.8) generates a holomorphic semigroup (see e.g. [32]). Therefore, the solution $u(t)$ of (1.8) is analytic with respect to $t$ for all $t>0$. However, due to the non-smoothness of the initial data, the solution may be singular at $t=0$. The aim of this section is to show how, by the use of socalled geometric time partitions and linearly increasing polynomial degrees in the time discretization, the low regularity of the solution at $t=0$ can be resolved.

Definition 3 A partition $\mathcal{M}_{M, \gamma}=\left\{J_{m}\right\}_{m=1}^{M}$ in $J=(0, T), T>0$, is called geometric with $M$ time steps $J_{m}=\left(t_{m-1}, t_{m}\right), m=1,2, \ldots, M$, and grading factor $\gamma \in(0,1)$, if

$$
t_{0}=0, \quad t_{m}=T \gamma^{M-m}, \quad 1 \leq m \leq M .
$$

Definition 4 A polynomial degree vector $\underline{r}$ is called linear with slope $\mu>0$ on $\mathcal{M}_{M, \gamma}$, if $r_{1}=0$ and $r_{m}=\lfloor\mu m\rfloor, m=2, \ldots, M$, where $\lfloor\mu m\rfloor=\max \left\{q \in \mathbb{N}_{0}: q \leq\right.$ $\mu m\}$. 
As in [23] we obtain the following a priori error estimate on the fully discrete (perturbed) dG method.

Theorem 8 Let $\rho \in(0,2]$, and $u$ be the solution of the parabolic problem (1.8) on $J \times I=(0, T) \times(a, b)$, with initial data $u_{0} \in \mathcal{V}_{\theta}$, for some $0<\theta \leq 1$. Let the assumptions of Theorem 7 be satisfied. Moreover, let (6.20)-(6.24) hold.

Then, there exist $\mu_{0}, m_{0}>0$ such that for all geometric partitions $\mathcal{M}_{M, \gamma}$ (cf. Definition 3) with $0<\gamma<1$ and $M \geq m_{0}|\log h|$, and all polynomial degree vectors $\vec{r}$ on $\mathcal{M}_{M, \gamma}$ (cf. Definition 4) with slope $\mu>\mu_{0}$, the fully discrete solution $\widetilde{U}_{h}^{d G}$ to (1.8) obtained by (6.26) on the finite element space $\mathcal{S}-\left(\mathcal{M}_{M, \gamma}, V_{h}\right)$ satisfies the a priori error estimate

$$
\left\|u(T)-\widetilde{U}_{h}^{d G}(T)\right\|_{L^{2}(I)} \leq C T^{-\frac{p+1}{\bar{m}}} h^{p+1} .
$$

Here, $h$ and $p$ are the mesh size and the polynomial degree of the spatial discretization, respectively, $C>0$ is a constant independent of $h$, and $\bar{m}$ is as in (2.4).

\subsection{Iterative solution of the linear systems}

The $\mathrm{dG}$ time stepping scheme (6.26) requires the solution of a nonsymmetric linear system in each time step. These systems can be solved approximately by incomplete GMRES iteration without loss in convergence rates in the error estimate (6.29). Based on the norm equivalences $(5.15)$, it can be shown as in $[23,26]$ that the overall complexity of this fully discrete $\mathrm{dG}$ time stepping is linear (up to some logarithmic terms). We indicate the argument and state the main result.

The dG time stepping scheme (6.28) requires a linear system of size $\left(r_{m}+1\right) N^{L}$ to be solved in each time step $m=1,2, \ldots, M$. Here and in what follows, in order to clarify the dependence on the refinement level $L$ explicitly, we denote by $N^{L}$ the dimension of the finite element space $V_{h}=V^{L}$.

For $1 \leq m \leq M$, let $\left\{\phi_{j}=\sqrt{j+\frac{1}{2}} L_{j}\right\}_{j=0}^{r_{m}}$, where $L_{j}$ is the $j$-th Legendre polynomial on $(-1,1)$ (normalized such that $L_{j}(1)=1$ ), be a basis of the polynomial space $\mathcal{P}_{r_{m}}(-1,1)$. Then, the temporal shape functions on the time interval $J_{m}$ are given by $\phi_{j} \circ F_{m}^{-1}$, where the mapping $F_{m}:(-1,1) \rightarrow J_{m}$ is given by

$$
t=F_{m}(\hat{t})=\frac{1}{2}\left(t_{m-1}+t_{m}\right)+\frac{1}{2} k_{m} \hat{t}, \quad k_{m}=t_{m}-t_{m-1}, \hat{t} \in(-1,1) .
$$

Writing $\widetilde{U}_{h, m}^{d G}(x, t)=\left.\widetilde{U}_{h}^{d G}\right|_{J_{m}}(x, t)$ and $W_{m}=\left.W\right|_{J_{m}}$ in (6.28) as

$$
\begin{aligned}
\widetilde{U}_{h, m}^{d G}(x, t) & =\sum_{j=0}^{r_{m}} \widetilde{U}_{h, m, j}^{d G}(x)\left(\phi_{j} \circ F_{m}^{-1}\right)(t), \\
W_{m}(x, t) & =\sum_{j=0}^{r_{m}} W_{m, j}(x)\left(\phi_{j} \circ F_{m}^{-1}\right)(t),
\end{aligned}
$$


the variational formulation (6.28) reads: find $\left(\tilde{U}_{h, m, j}^{d G}\right)_{j=0}^{r_{m}} \in\left(V_{h}\right)^{\underline{r}}$ such that there holds for all $\left(W_{m, i}\right)_{i=0}^{r_{m}} \in\left(V_{h}\right)^{r}$,

$$
\begin{aligned}
& \sum_{i, j=0}^{r_{m}} C_{i j}\left(\widetilde{U}_{h, m, j}^{d G}, W_{m, i}\right)_{L^{2}(I)}+\frac{k_{m}}{2} \sum_{i=0}^{r_{m}} \tilde{a}\left(\widetilde{U}_{h, m, j}^{d G}, W_{m, i}\right) \\
& \quad=\sum_{i=0}^{r_{m}} f_{m, i}\left(W_{m, i}\right)
\end{aligned}
$$

where, for $i, j=0,1, \ldots, r_{m}$,

$$
C_{i j}=\sigma_{i j} \sqrt{\left(i+\frac{1}{2}\right)\left(j+\frac{1}{2}\right)}, \quad \sigma_{i j}= \begin{cases}(-1)^{i+j} & \text { if } j>i \\ 1 & \text { else }\end{cases}
$$

and $f_{m, i}(v)=\phi_{i}(-1)\left(\tilde{U}_{h, m-1}^{d G-}\left(t_{m-1}\right), v\right)$.

From now on, for the sake of readability, we will drop the subscript $m$. Then, denoting by $\mathbf{M}$ and $\widetilde{\mathbf{A}}$ the mass and (compressed) stiffness matrix on $V^{h}=V^{L}$ with respect to $(\cdot, \cdot)$ and $\widetilde{a}(\cdot, \cdot)$, respectively, (6.28) takes the matrix form

$$
\mathbf{R} \underline{u}=\underline{f} \quad \text { with } \mathbf{R}=\mathbf{C} \otimes \mathbf{M}+\frac{k}{2} \mathbf{I} \otimes \tilde{\mathbf{A}},
$$

where $\underline{u}$ denotes the coefficient vector of $\widetilde{U}_{h, m}^{d G}=\left.\widetilde{U}_{h}^{d G}\right|_{J_{m}} \in \mathcal{P}_{r_{m}}\left(J_{m}, V_{h}\right)$.

In [26] it has been found that the system (6.31) of size $(r+1) N^{L}$ can be reduced to solving $r+1$ linear systems of size $N^{L}$. To this end, let $\mathbf{C}=\mathbf{Q T Q}^{\top}$ be the Schur decomposition of the $(r+1) \times(r+1)$ matrix $\mathbf{C}$ with a unitary matrix $\mathbf{Q}$ and an upper triangular matrix $\mathbf{T}$. Note that the diagonal of $\mathbf{T}$ contains the eigenvalues $\lambda_{1}, \lambda_{2}, \ldots, \lambda_{r+1}$ of $\mathbf{C}$. Multiplying (6.31) by $\mathbf{Q}^{\top} \otimes \mathbf{I}$ from the left results in the linear system

$$
\left(\mathbf{T} \otimes \mathbf{M}+\frac{k}{2} \mathbf{I} \otimes \tilde{\mathbf{A}}\right) \underline{w}=\underline{g} \quad \text { with } \underline{w}=\left(\mathbf{Q}^{\top} \otimes \mathbf{I}\right) \underline{u}, \underline{g}=\left(\mathbf{Q}^{\top} \otimes \mathbf{I}\right) \underline{f} .
$$

This system is block-upper-triangular. With $\underline{w}=\left(\underline{w}_{0}, \underline{w}_{1}, \ldots, \underline{w}_{r}\right), \underline{w}_{j} \in \mathbb{C}^{N^{L}}$, we obtain its solution by solving

$$
\left(\lambda_{j+1} \mathbf{M}+\frac{k}{2} \widetilde{\mathbf{A}}\right) \underline{w}_{j}=\underline{s}_{j}
$$

for $j=r, r-1, \ldots, 0$, where $\underline{s}_{j}=\underline{g}_{j}-\sum_{l=j+1}^{r} \mathbf{T}_{j+1, l+1} \mathbf{M} \underline{w}_{l}$.

By (6.32), a dG-time step of order $r \geq 0$ amounts to solving $r+1$ linear systems of the form

$$
\mathbf{B}=\lambda \mathbf{M}+\frac{k}{2} \tilde{\mathbf{A}}
$$

Here, $\lambda$ is an eigenvalue of $\mathbf{C}$ defined in (6.30). Estimates on the eigenvalues of $\mathbf{C}$ have been established in [26]. 
For the preconditioning of (6.33), we define the matrix $\mathbf{S}$ and the scaled matrix $\widehat{\mathbf{B}} \in \mathbb{R}^{N^{L}} \times \mathbb{R}^{N^{L}}$ by

$$
\mathbf{S}=\left(\operatorname{Re}(\lambda) \mathbf{I}+\frac{k}{2} \mathbf{D}^{m(x)}\right)^{\frac{1}{2}}, \quad \widehat{\mathbf{B}}=\mathbf{S}^{-1} \mathbf{B S}^{-1},
$$

where $\mathbf{D}^{m(x)}$ is defined in (5.12).

The preconditioned linear equations corresponding to (6.32) are solved approximately with incomplete $\operatorname{GMRES}\left(m_{0}\right)$ iteration (restarted every $m_{0} \geq 1$ iterations). There holds:

Theorem 9 Let the assumptions of Theorem 8 hold. Then, choosing the number and order of time steps such that $M=r=\mathcal{O}(|\log h|)$ and in each time step $n_{G}=\mathcal{O}(|\log h|)^{5}$ GMRES iterations,

$$
\left\|u(T)-\widehat{U}_{h}^{d G}(T)\right\|_{L^{2}(I)} \leq C T^{-\frac{p+1}{\bar{m}}} h^{p+1},
$$

where $\widehat{U}_{h}^{d G}$ denotes the (perturbed) $d G$ approximation of the exact solution $u$ to (1.8) obtained by the incomplete GMRES $\left(m_{0}\right)$ method.

Applying the matrix compression techniques from Sect. 5, the judicious combination of geometric mesh refinement and linear increase of polynomial degrees in the dG time-stepping scheme (Theorem 8), and an appropriate number of GMRES iterations (Theorem 9), results in an linear (up to some logarithmic terms) overall complexity of the fully discrete scheme (6.26) for the solution of the parabolic problem (1.8).

Theorem 10 Under the assumptions of Theorems 8 and 9, the fully discrete scheme (6.26) with $n_{G}=\mathcal{O}(|\log h|)^{5}$ GMRES $\left(m_{0}\right)$ iterations per time step yields $\widehat{U}_{h}^{d G}(T)$ in at most $\mathcal{O}\left(N(\log N)^{8}\right)$ operations, where $N=N^{L}=\operatorname{dim} V_{h}=\mathcal{O}\left(h^{-1}\right)$ is the number of spatial degrees of freedom.

\section{Numerical examples}

In this section we describe the implementation of one dimensional Feller processes using the techniques described above. Assume the risk-neutral dynamics of the underlying asset to be given by

$$
S_{t}=S_{0} e^{r t+X_{t}}
$$

where $X$ is a Feller process with characteristic triple $(\gamma(x), \sigma(x), N(x, d y))$ under a risk neutral measure $\mathbb{Q}$ such that $e^{X}$ is a martingale with respect to the canonical filtration of $X$. In the following we set $r=0$ for notational convenience. We will only consider Feller processes $X$ that are admissible market models:

Definition 5 A Feller process $X$ with characteristic triple $(\gamma(x), \sigma(x), N(x, d y))$ is called an admissible market model if 
1. There exist constants $\beta^{-}, C>0$ and $\beta^{+}>1$ independent of $x$ s.t. for $N(x, d y)=$ $k(x, y) \mathrm{d} y$

$$
k(x, y) \leq C \begin{cases}e^{-\beta^{-}|y|}, & y<-1 \\ e^{-\beta^{+} y}, & y>1\end{cases}
$$

2. There exists a constant $C$ independent of $x$ s.t.

$$
\frac{1}{2}(k(x, y)+k(x,-y)) \geq C \frac{1}{|y|^{2 m(x)}}, \quad 0<|y| \leq 1, x \in \mathbb{R} .
$$

3. There exist a function $m(x) \in C^{\infty}(\mathbb{R})$, constants $\rho, \delta$ and $c$ s.t. $m(x) \in(0,1)$, $0 \leq \delta \leq \rho \leq 1$ and

$$
\left|D_{x}^{\beta} D_{y}^{\alpha} k(x, y)\right| \leq c \alpha ! \beta !|y|^{-1-2 m(x)-\alpha \rho-\beta \delta} \quad \forall \alpha, \beta \in \mathbb{N}_{0}, y \neq 0 .
$$

Remark 2 Condition (2) implies the sector condition (6.11) and condition (3) implies $a(x, \xi) \in S_{\rho, \delta}^{2 m(x)}$.

The next lemma shows how the martingale condition on $e^{X}$ can be expressed in terms of the characteristic triple.

Lemma 14 Let $X$ be an admissible market model with characteristic triple $(\gamma(x), \sigma(x), N(x, d y))$. Then $e^{X}$ is a $\mathbb{Q}$ martingale with respect to the canonical filtration of $X$ if and only if

$$
\frac{\sigma(x)^{2}}{2}+\gamma(x)+\int_{\mathbb{R}}\left(e^{y}-1-y\right) N(x, d y)=0 \quad \forall x \in \mathbb{R} .
$$

Proof For the proof of this lemma we cannot use standard arguments as e.g. in [28], since stationarity of the increments is required. We consider the characteristic function of the random variable $X_{t}-x$ as in [15], i.e.

$$
\lambda_{t}(x, \xi)=E^{x}\left(e^{i\left(X_{t}-x\right) \xi}\right) .
$$

The martingale condition implies

$$
0=\lambda_{t}(x,-i)-\lambda_{0}(x,-i) \quad \forall x \in \mathbb{R}, \forall t \in \mathbb{R}_{+} .
$$

The right-hand side can be written as

$$
\begin{aligned}
\lambda_{t}(x,-i)-\lambda_{0}(x,-i) & =\left.t \int_{0}^{1} \frac{d}{d s} \lambda_{s}(x,-i)\right|_{s=\eta t} d \eta \\
& =t \int_{0}^{1} \frac{d}{d s}\left(\left.e^{-x} T_{s}\left(e^{(\cdot)}\right)(x)\right|_{s=\eta t}\right) d \eta \\
& =-t \int_{0}^{1} e^{-x} T_{t \eta}\left(e^{(\cdot)} a(\cdot,-i)\right)(x) d \eta,
\end{aligned}
$$


where we have used $(d / d s) T_{s} u=T_{s} A u$ and $A\left(e^{i(\cdot, \xi)}\right)=-e^{i x \xi} a(x, \xi)$. This implies $a(x,-i)=0$. The reverse implication follows analogously.

We now consider problem (1.8) with $A$ being the infinitesimal generator of an admissible martingale market model. The corresponding PIDE for a sufficiently smooth payoff $g$ in time-to-maturity reads:

$$
\begin{aligned}
& \frac{\partial u}{\partial t}-\mathcal{A}_{\mathrm{LV}}-\mathcal{A}_{\mathrm{J}}=0 \quad \text { in }(0, T) \times \mathbb{R} \\
& u(0, x)=g(x) .
\end{aligned}
$$

The local volatility operator $\mathcal{A}_{\mathrm{LV}}$ and the jump operator $\mathcal{A}_{\mathrm{J}}$ are given as:

$$
\begin{aligned}
\mathcal{A}_{\mathrm{LV}}(\varphi) & =\frac{\sigma(x)^{2}}{2} \frac{\partial^{2} \varphi}{\partial x^{2}}+\gamma(x) \frac{\partial \varphi}{\partial x} \\
\mathcal{A}_{\mathrm{J}}(\varphi) & =\int_{\mathbb{R}}\left(\varphi(x+y)-\varphi(x)-y \partial_{x} \varphi(x)\right) k(x, y) d y .
\end{aligned}
$$

We localize the above problem to a bounded domain $I$ and obtain the following weak formulation: find $u_{I} \in L^{2}((0, T), V) \cap H^{1}\left((0, T), V^{*}\right)$ such that

$$
\begin{aligned}
& \left(\partial_{t} u_{I}, v\right)+a\left(u_{I}, v\right)=0, \quad \forall v \in V, \text { a.e. in }[0, T], \\
& u_{I}(0)=g,
\end{aligned}
$$

where the bilinear form $a$ is given for $\phi, \psi \in C_{0}^{\infty}(I)$ by

$$
\begin{aligned}
a(\phi, \psi) & =a_{\mathrm{LV}}(\widetilde{\phi}, \widetilde{\psi})+a_{\mathrm{J}}(\widetilde{\phi}, \widetilde{\psi}), \\
a_{\mathrm{LV}}(\widetilde{\phi}, \widetilde{\psi}) & =\frac{1}{2}\left(\sigma(x)^{2} \widetilde{\phi}(x)^{\prime}, \widetilde{\psi}(x)^{\prime}\right)-\left(\widetilde{\gamma}(x) \widetilde{\phi}(x)^{\prime}, \widetilde{\psi}(x)\right), \\
a_{\mathrm{J}}(\widetilde{\phi}, \widetilde{\psi}) & =-\int_{\mathbb{R}} \int_{\mathbb{R}}\left(\widetilde{\phi}(x+y)-\widetilde{\phi}(x)-y \partial_{x} \widetilde{\phi}(x)\right) \widetilde{\psi}(x) k(x, y) d y d x .
\end{aligned}
$$

Note that, unlike in the Black-Scholes case, the non-local operator $\mathcal{A}_{\mathrm{J}}$ forces to specify the test functions outside of $I$. The function $\tilde{\gamma}(x)$ is given as $\tilde{\gamma}(x)=$ $\gamma(x)+\sigma(x) \sigma^{\prime}(x)$ while $\widetilde{\phi}$ and $\widetilde{\psi}$ are the zero extensions of $\phi$ and $\psi$ outside of a bounded interval $I=(a, b)$. The space $V$ in the above definition equals $H_{0}^{1}(I)$ if the diffusion is non-trivial and $\tilde{H}^{m(x)}(I)$ in the pure jump case. Existence and uniqueness of the solution have been proven above in either cases. We restrict ourselves to the pure jump case, as the discretization of the diffusion operator is standard.

The bilinear form $a_{\mathrm{J}}$ is not easy to discretize, as the integration kernel $k(x, y)$ might have a singularity of order up to 3 at $y=0$.

Therefore, we use partial integration in $y$ twice and the regularity of $\phi$ to obtain a weakly singular integration kernel, i.e.

$$
a_{\mathrm{J}}(\tilde{\phi}, \widetilde{\psi})=-\int_{\mathbb{R}} \int_{\mathbb{R}} \widetilde{\phi}^{\prime \prime}(x+y) \widetilde{\psi}(x) k^{(-2)}(x, y) d y d x,
$$


where $k^{(-n)}(x, y)$ denotes the $n$-th antiderivative in $y$ :

$$
k^{(-n)}(x, y)= \begin{cases}\int_{-\infty}^{y} k^{(-n+1)}(x, z) d z & \text { if } z<0 \\ -\int_{y}^{\infty} k^{(-n+1)}(x, z) d z & \text { if } z>0\end{cases}
$$

Finally we use partial integration with respect to $x$ to get:

$$
\begin{aligned}
a_{\mathrm{J}}(\tilde{\phi}, \widetilde{\psi})= & \int_{\mathbb{R}} \int_{\mathbb{R}} \widetilde{\phi}^{\prime}(x+y) \widetilde{\psi}^{\prime}(x) k^{(-2)}(x, y) d y d x \\
& +\int_{\mathbb{R}} \int_{\mathbb{R}} \widetilde{\phi}^{\prime}(x+y) \widetilde{\psi}(x) k_{x}^{(-2)}(x, y) d y d x .
\end{aligned}
$$

Remark 3 The major differences to the Lévy case reside in the second term of (7.3) and the $x$-dependence of the integration kernels. In order for the described procedure to be feasible, the second antiderivatives of the jump kernel need to be available. This is the case for many processes such as CGMY-type or Variance Gamma-type Feller processes, for example.

The calculation of the stiffness matrices $A$ and $\widetilde{A}$ involves integration over twodimensional domains with integration kernels that might be weakly singular on a diagonal of the domain or in one of the corners. In most cases we have to rely on numerical methods to obtain the matrix entries, for example by using composite Gauss quadrature rules as in [4] or [36, Chap. 5], which leads to exponential convergence in the number of quadrature points.

In the following we will consider a special family of Feller processes to confirm the theoretical results of the previous chapters.

Example 1 We consider a CGMY-type Feller process with jump kernel

$$
k(x, y)=C\left\{\begin{array}{ll}
e^{-\beta^{+} y} y^{-1-\alpha(x)}, & y>0 \\
e^{-\beta^{-}|y|}|y|^{-1-\alpha(x)}, & y<0,
\end{array} \quad \alpha(x)=k e^{-x^{2}}+0.5\right.
$$

This process has no Gaussian component and the drift $\gamma(x)$ is chosen according to (7.1).

We will also consider the following family of processes that do not fulfill the conditions of the theory developed above, since the variable order is assumed to be Lipschitz continuous only.

Example 2 We consider again a CGMY-type Feller process with jump kernel

$$
k(x, y)=C \begin{cases}e^{-\beta^{+} y} y^{-1-\alpha(x)}, & y>0, \\ e^{-\beta^{-}|y|}|y|^{-1-\alpha(x)}, & y<0\end{cases}
$$




$$
\alpha(x)=0.5+k \begin{cases}0.4 x, & 0.25>x>0, \\ 0.8 x-0.1, & 0.5>x \geq 0.25, \\ -0.4 x+0.5, & 0.75>x \geq 0.5 \\ -0.8 x+0.8, & 1>x \geq 0.75, \\ 0.5, & \text { else. }\end{cases}
$$

This process has no Gaussian component and the drift $\gamma(x)$ is chosen according to (7.1).

In Fig. 1 the stiffness matrix for the process in Example 1 is depicted. In a next step we study the number of non-zero entries of the uncompressed and compressed stiffness matrix. Due to Proposition 5 we expect essentially linear growth of the number of non-zero elements for the compressed matrix (Fig. 2).

The condition numbers of the preconditioned stiffness matrices have to be uniformly bounded in the number of levels due to Theorem 4. A parameter study for various choices of $k$ in Example 1 and Example 2 is shown in Fig. 3. The condition numbers are uniformly bounded and of order $10^{1}$ in most cases, although the result from Theorem 4 only applies to Example 1 . For variable orders with $1.95 \leq \bar{\alpha}$ we obtain condition numbers of order $10^{2}$. Note that the condition numbers are not only influenced by the order of the singularity of the jump kernel at $z=0$, but also by the rates of exponential decay $\beta^{+}$and $\beta^{-}$. Fast decaying tails, i.e. large $\beta^{+}$and $\beta^{-}$may lead to larger constants.

Figure 4 shows the price of a European put option for several Lévy processes and one Feller process. In the Feller case we choose $\alpha(x)=0.8 e^{-x^{2}}+0.1$ in Example 1 and for the Lévy models we set $Y=[0.1,0.5,0.7,0.8,0.9]$. In all cases we set $C=1$, $\beta^{+}=\beta^{-}=10$ and use truncation parameters $a=-3, b=3$ in log-moneyness coordinates. The prices in the Feller model are significantly different from the prices in the different Lévy models. This can be explained by the ability of the Feller model

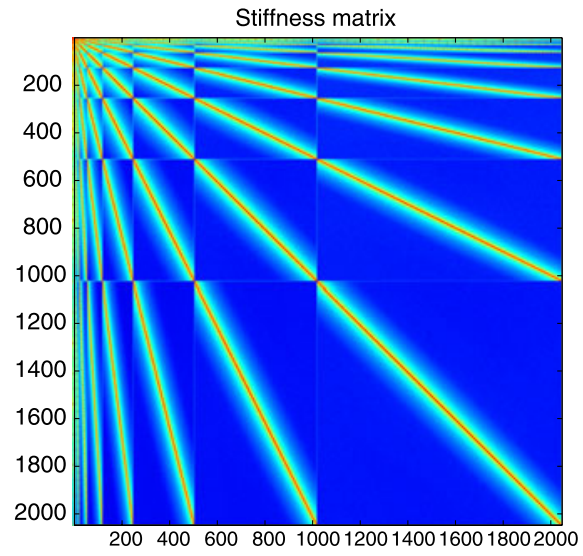

(a) Stiffness matrix

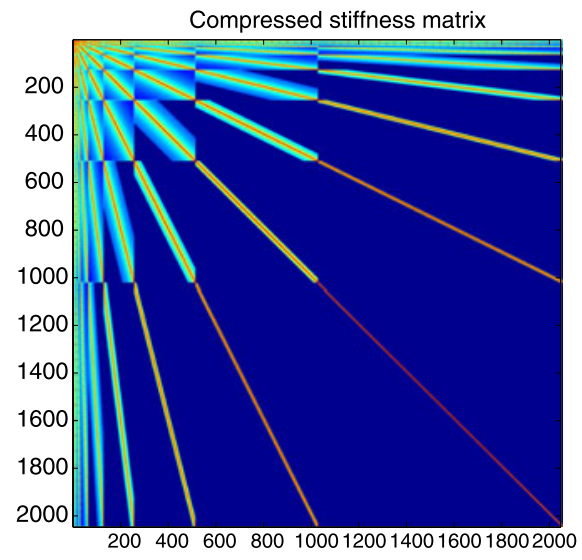

(b) Compressed stiffness matrix

Fig. 1 Stiffness matrices for the pure jump case with CGMY-type Lévy kernel $\left(Y(x)=1.25 e^{-x^{2}}+0.5\right)$ 


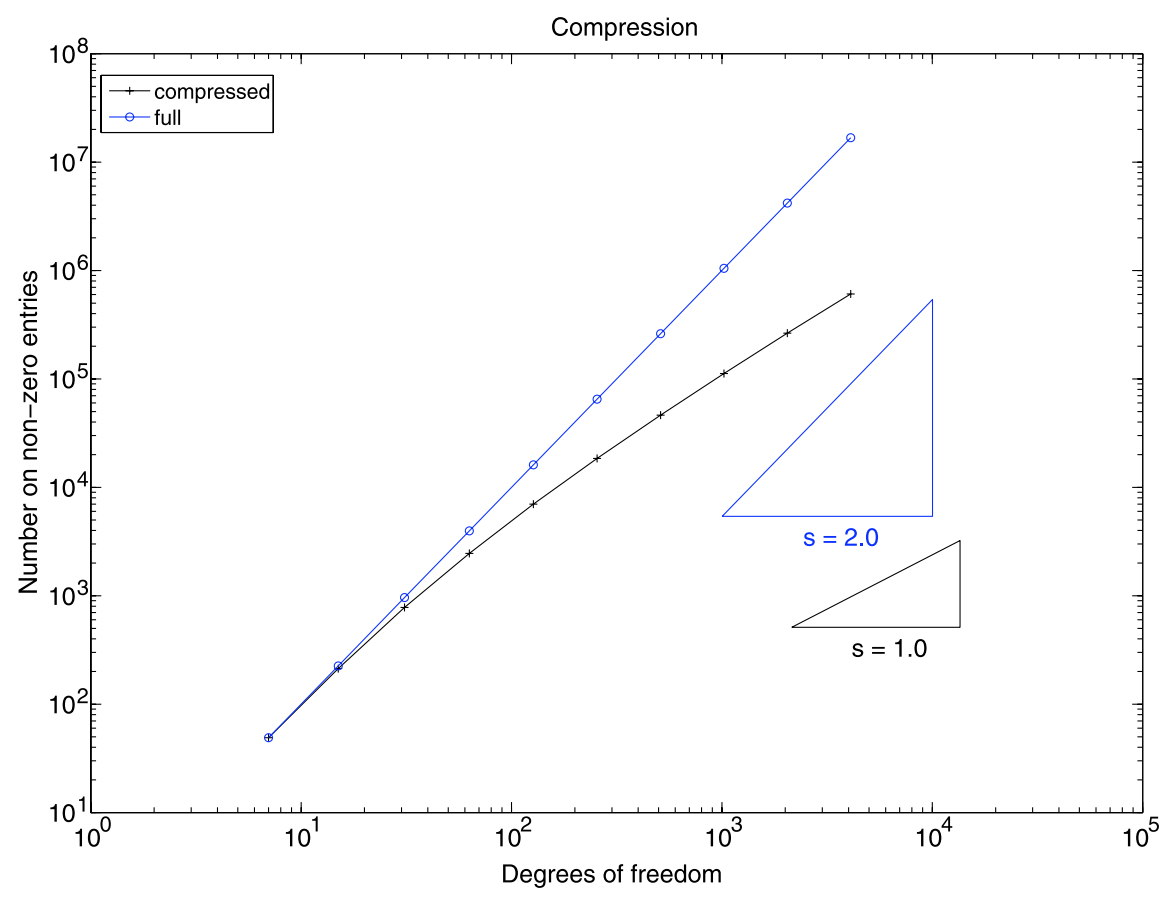

Fig. 2 Number of non-zero entries of the compressed/uncompressed stiffness matrix versus number of degrees of freedom corresponding to the Lévy kernel in Example 1 and $k=1.25$

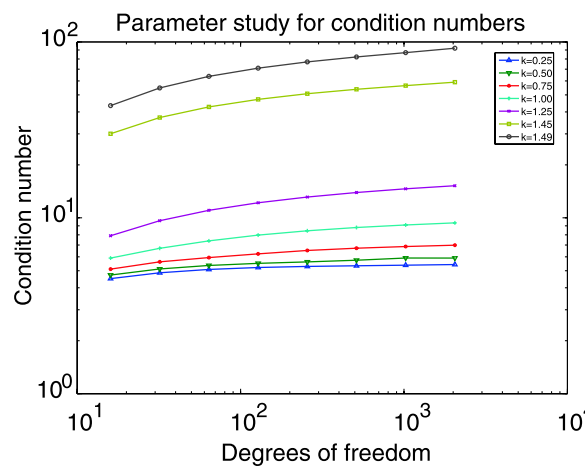

(a) Example 1

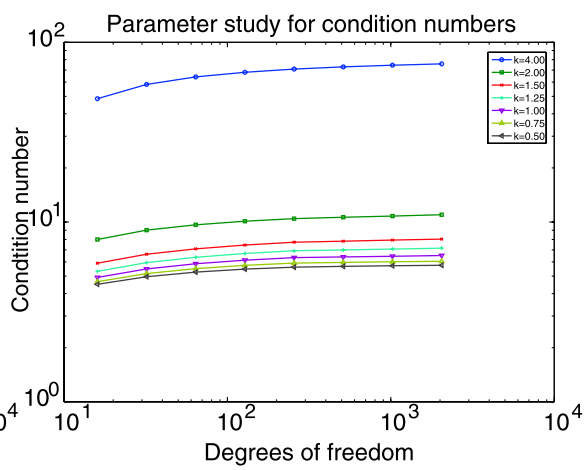

(b) Example 2

Fig. 3 Condition numbers for different levels and choices of $k$

to account for different tail behavior for different states of the process, which is not possible using Lévy processes. 


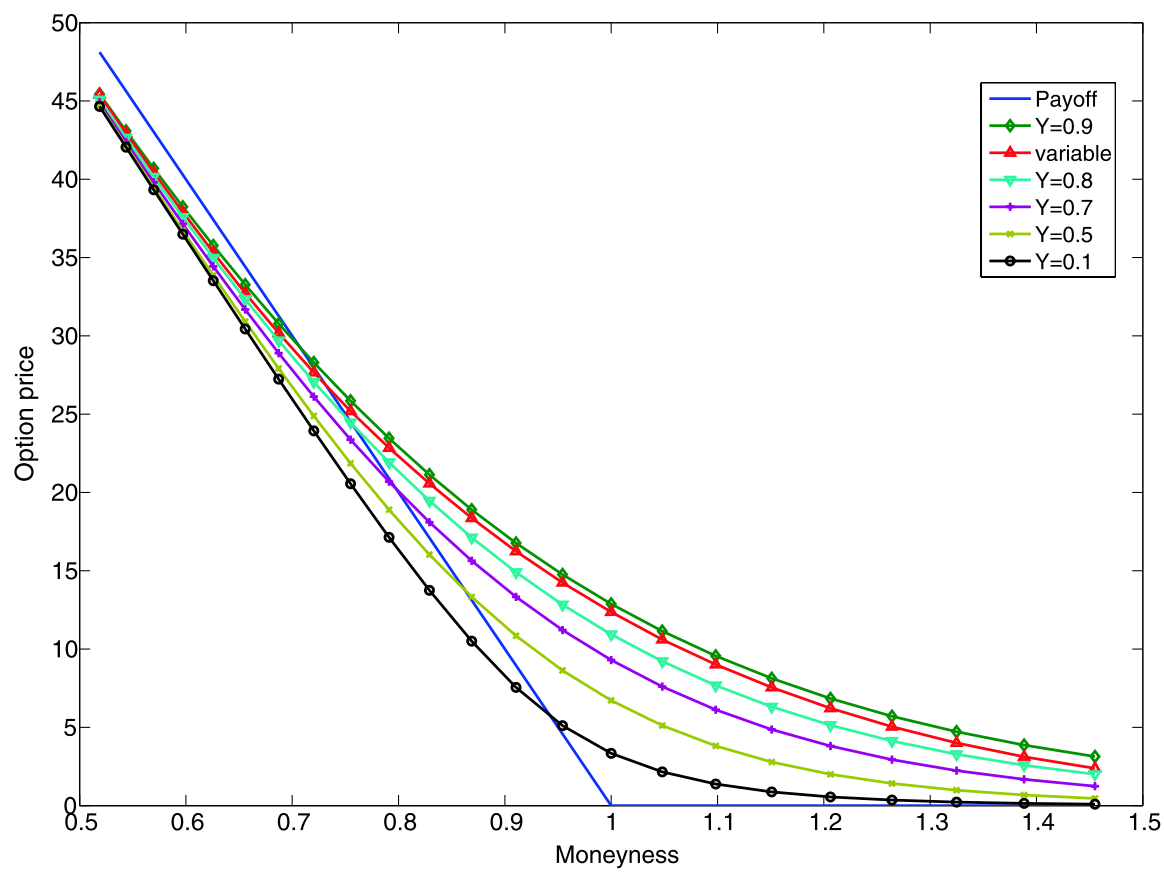

Fig. 4 Option prices for several models for a European put option with $T=1$ and $K=100$

\section{References}

1. Bass, R.F.: Uniqueness in law for pure jump Markov processes. Probab. Theory Relat. Fields 79, 271-287 (1988)

2. Bertoin, J.: Lévy Processes. Cambridge University Press, Cambridge (1996)

3. Black, F., Scholes, M.: The pricing of options and corporate liabilities. J. Polit. Econ. 81, 637-654 (1973)

4. Chernov, A., von Petersdorff, T., Schwab, C.: Exponential convergence of hp quadrature for integral operators with Gevrey kernels. Research-Report No. 2009-03, SAM, ETH

5. Cohen, A.: Numerical Analysis of Wavelet Methods. Studies in Mathematics and its Applications, vol. 32. North Holland-Elsevier, Amsterdam (2003)

6. Cont, R., Tankov, P.: Financial Modelling with Jump Processes. Chapman and Hall/CRC Press, London (2004)

7. Courrège, Ph.: Sur la forme intégro-differentielle des opérateurs de $C_{K}^{\infty}$ dans $C$ satisfaisant du principe du maximum. Sém. Théor. Potentiel 38 (1965-1966)

8. Dahmen, W., Schneider, R.: Wavelets with complementary boundary conditions, Function spaces on the cube. Results Math. 34, 255-293 (1998)

9. Delbaen, F., Schachermayer, W.: The variance-optimal martingale measure for continuous processes. Bernoulli 2, 81-105 (1996)

10. Dijkema, T.J.: Adaptive tensor product wavelet methods for solving PDEs. Dissertation, University of Utrecht (2009)

11. Harbrecht, H., Stevenson, R.: Wavelets with patchwise cancellation properties. Math. Comput. 75, 1871-1889 (2006)

12. Hoh, W.: Pseudodifferential operators with negative definite symbols of variable order. Rev. Math. Iberoam. 16, 219-241 (2000)

13. Jacob, N.: Pseudo-Differential Operators and Markov Processes. Vol. 1: Fourier Analysis and Semigroups. Imperial College Press, London (2001) 
14. Jacob, N.: Pseudo-Differential Operators and Markov Processes. Vol. 2: Generators and their Potential Theory. Imperial College Press, London (2002)

15. Jacob, N.: Characteristic functions and symbols in the theory of feller processes. Potential Anal. 6, 61-68 (1998)

16. Kikuchi, K., Negoro, A.: On Markov Process generated by pseudodifferential operator of variable order. Osaka J. Math. 34, 319-335 (1997)

17. Kumano-go, H.: Pseudo-Differential Operators. MIT Press, Cambridge (1981)

18. Leopold, H.G.: On function spaces of variable order of differentiation. Forum Math. 3, 1-21 (1991)

19. Lions, J.L., Magenes, E.: Non-Homogeneous Boundary Value Problems and Applications. Springer, Berlin (1972)

20. Matache, A.-M., Nitsche, P.A., Schwab, C.: Wavelet Galerkin pricing of American contracts on Lévy driven assets. Quant. Finance 5, 403-424 (2005)

21. Matache, A.-M., von Petersdorff, T., Schwab, C.: Fast deterministic pricing of options on Lévy driven assets. Math. Model. Numer. Anal. 38, 37-71 (2004)

22. Matache, A.-M., Schwab, C., Wihler, T.P.: Fast numerical solution of parabolic integro-differential equations with applications in finance. SIAM J. Sci. Comput. 27, 369-393 (2005)

23. Matache, A.-M., Schwab, C., Wihler, T.P.: Linear complexity solution of parabolic integrodifferential equations. Numer. Math. 104, 69-102 (2006)

24. Merton, R.C.: Option pricing when the underlying stocks are discontinuous. J. Financ. Econ. 5, 125144 (1976)

25. von Petersdorff, T., Schwab, C.: Wavelet discretizations of parabolic integro-differential equations. SIAM J. Numer. Anal. 41, 159-180 (2003)

26. von Petersdorff, T., Schwab, C.: Numerical solution of parabolic equations in high dimensions. Math. Model. Numer. Anal. 38, 93-128 (2004)

27. Primbs, M.: Stabile biorthogonale Spline-Waveletbasen auf dem Intervall. Dissertation, University Duisburg-Essen (2006)

28. Reich, N., Schwab, C., Winter, C.: On Kolmogorov equations for anisotropic multivariate Lévy processes. Research Report No. 2008-03, SAM, ETH

29. Sato, K.-I.: Lévy Processes and Infinitely Divisible Distributions. Cambridge University Press, Cambridge (1999)

30. Schilling, R.: On the existence of Feller processes with a given generator. Preprint (2004). http://www.math.tu-dresden.de/sto/schilling/sources/papers/existenz.pdf

31. Schneider, R.: Multiskalen- und Wavelet-Matrixkompression. Analysisbasierte Methoden zur effizienten Lösung grosser vollbesetzter Gleichungssysteme. Advances in Numerical Mathematics. Teubner, Stuttgart (1998)

32. Schötzau, D., Schwab, C.: $h p$-discontinuous Galerkin time-stepping for parabolic problems. C. R. Acad. Sci. Paris 333, 1121-1126 (2001)

33. Shubin, M.A.: Pseudodifferential Operators and Spectral Theory. Springer, Berlin (1985)

34. Stein, E.M.: Harmonic Analysis. Princeton University Press, Princeton (2002)

35. Urban, K.: Wavelet Methods for Elliptic Partial Differential Equations. Oxford University Press, Oxford (2009)

36. Winter, C.: Wavelet Galerkin schemes for option pricing in multidimensional Lévy models. Dissertation No. 18221, ETH (2009) 